\title{
Liquid-Vapor Phase Equilibrium in Solutions of Oxygen and Nitrogen at Pressures Below One Atmosphere ${ }^{1}$
}

\author{
George T. Armstrong, Jack M. Goldstein, ${ }^{2}$ and D. Ellis Roberts
}

\begin{abstract}
A cryostat and equilibrium vessel, together with auxiliary apparatus for establishing equilibrium between liquid and vapor phases of solutions of low boiling materials by a circulation method, is described. The equilibrium vessel incorporates a novel liquid sampling device. Vapor and liquid compositions and total vapor pressures of solutions of oxygen and nitrogen were measured along isotherms at $77.5^{\circ}, 70^{\circ}$ and $65^{\circ} \mathrm{K}$. The activity coefficients of nitrogen and oxygen may be represented by equations of the form

$$
\frac{R T}{V_{\mathrm{N}_{2}}} \log _{e} \gamma_{\mathrm{N}_{2}}=A_{12} \phi_{\mathrm{O}_{2}}^{2} \text { and } \frac{R T}{V_{\mathrm{O}_{2}}} \log _{e} \gamma_{\mathrm{O}_{2}}=A_{12} \phi_{\mathrm{N}_{2}}^{2}
$$

in which $A_{12}$ in $\mathrm{cal} / \mathrm{cm}^{3}$ mole has the values 1.22 at $77.5^{\circ}, 1.38$ at $70^{\circ}$, and 1.47 at $65^{\circ} \mathrm{K}$. The deviations of the solutions from ideality are much less than is to be expected of regular solutions, in which the interaction energy between unlike molecules follows a geometric mean law. The data are not entirely consistent with the assumption that molar volumes are additive in the solutions.
\end{abstract}

\section{Introduction}

Vapor-liquid equilibrium measurements have been made on the system oxygen-nitrogen over isotherms covering most of the range from $75^{\circ} \mathrm{K}$ to the critical region. Baly [1] ${ }^{3}$ made measurements by a static method at one atmosphere only. Inglis [2] determined two isotherms, at $74.7^{\circ} \mathrm{K}$ and $79.07^{\circ} \mathrm{K}$ by a circulation method. Dodge and Dunbar [3] provided the most complete study of the system, by a circulation method determining isotherms at $90^{\circ}$, $100^{\circ}, 110^{\circ}, 120^{\circ}$, and $125^{\circ}$. Trapeznikova and Shubnikov [4] determined an isotherm at $85^{\circ} \mathrm{K}$. Sagenkahn and Fink [5] made a series of measurements by a static method in the region from one to two atmospheres. The chief interest in this system has been to provide information useful in the separation of air by fractional distillation, and this accounts for the preponderance of measurements at superatmospheric pressures.

The production of hypersonic gas velocities in wind tunnels creates temperatures in some cases below $75^{\circ} \mathrm{K}$. This development has created an interest in the phase behavior of the components of air at temperatures well below those for which data exist. The present research was undertaken to fill the uncertainties in the liquid region below the normal boiling point of nitrogen.

The possibility that association of oxygen molecules into dimers might occur and cause large deviations from ideality offered an added incentive to undertake the study of this system.

This work was sponsored by the Air Research and Development Command, Department of the Air Force.

2 Present address, Department of Chemistry, University of Pennsylvania, Philadelphia, $\mathrm{Pa}$.

3 Figures in brackets indicate the literature references at the end of this paper.

\section{Experimental Apparatus and Procedures}

\subsection{General Experimental Plan}

The measurements were made by a circulation method. The vapor mixture from a room temperature reservoir was circulated by a positively acting pump through the liquid solution in a constant temperature vessel, and returned to the room temperature reservoir. Circulation was continued until equilibrium was established; the temperature and pressure were then measured, and samples of liquid and vapor were extracted for analysis.

\subsection{Equilibrium Vessel and Cryostat}

The equilibrium vessel is protected from external temperatures by two liquid refrigerant baths and an evacuated chamber. The equilibrium vessel is surrounded by a copper can, which can be highly evacuated, or filled with helium for heat transfer. The can not only encloses the vacuum space, but also, because of its high thermal conductivity, serves to present a uniform temperature environment to the equilibrium vessel. The equilibrium vessel in its thermal shield is immersed in a liquid nitrogen bath. This bath, consisting of a one-liter Dewar flask in a covered container suitable for evacuation, is maintained at the desired isothermal temperature, and will be called the isothermal bath. The isothermal bath cover is held by screws to a heavy flange on the rim of the container. The joint between the cylindrical container and the cover is sealed by a narrow teflon ring gasket. The cover is fitted with a $1 / 2$-in. outside diameter thin wall monel tube, which serves for filling and also for a vacuum pump connecting tube. 
In order to permit maintenance of the isothermal bath at the desired temperature for a reasonable length of time without excessive loss of liquid, it is in turn placed in an outer bath of liquid nitrogen boiling freely at the prevailing atmospheric pressure. A loose fitting lid covers the outer nitrogen bath and at the same time provides support for the isothermal bath, which hangs within. Losses of refrigerant from the outer bath are rather rapid, because of the large number of tubes entering it, and because it is necessary to have it filled nearly to the top, so that frequent refilling is necessary.

The inner isothermal bath, protected as it is on all sides by liquid nitrogen, requires much less attention. A filling can be expected to last $4 \mathrm{hr}$ or longer, even when operating at the lowest temperatures. The temperature of the isothermal bath is controlled by regulating the pressure under which the refrigerant (commercial liquid nitrogen) boils. The pressure is reduced by a large vacuum pump, and is maintained near a predetermined value by a cartesian-diver type manostat. The temperature control by this method suffers from two drawbacks. (1) The approach to thermal equilibrium within the isothermal bath is slow, so that a change in the manostat setting is not completely reflected in the temperature of the equilibrium vessel for an hour or more. (2) The constant pumping away of the liquid nitrogen from the isothermal bath continually enriches the residual liquid in oxygen, and causes a slow rise of the bath temperature. The effect of the first factor is to require some patience in the establishment of the manostat setting, while the second factor results in the final temperature setting of many of the points lying one or two tenths of a degree above the desired isotherm.

The details of the equilibrium vessel are shown in figure 1. It consists of a copper cylinder, D, with an inside diameter of 1 in., an inside height of about 3 in., and a wall thickness of $1 / 8$ in. The volume of the vessel is about $36 \mathrm{~cm}^{3}$.

The lid, E, of the equilibrium vessel is a disk of 1/4-in. brass, having a shallow shoulder cut around the rim. This fits a similar shoulder in a flange, F, on the top of the cylinder. A gasket, G, of 0.015-in. diameter fine gold wire forms a gas-tight joint when the lid is bolted to the flange.

Suspended from the lid is the thermometer well, $\mathrm{H}$, a thin wall copper tube, which snugly fits the small platinum resistance thermometer, J. Also suspended from the lid is a sheet copper basket, $\mathrm{K}$, of semicircular shape, so positioned that the thermometer well passes, with a small amount of clearance, through a hole in the bottom of the basket. The basket is placed in a position with respect to the vapor lift pump, L, such that liquid, forced by the circulating vapors through the lift pump, is caught in the basket and trickles down the thermometer well.

The circulating vapors, passing through a quarterinch tube, $\mathrm{M}$, enter the vapor Jift pump through a T joint at $\mathrm{N}$. The vapor lift pump is the tube, M, connecting the bottom of the equilibrium vessel to a

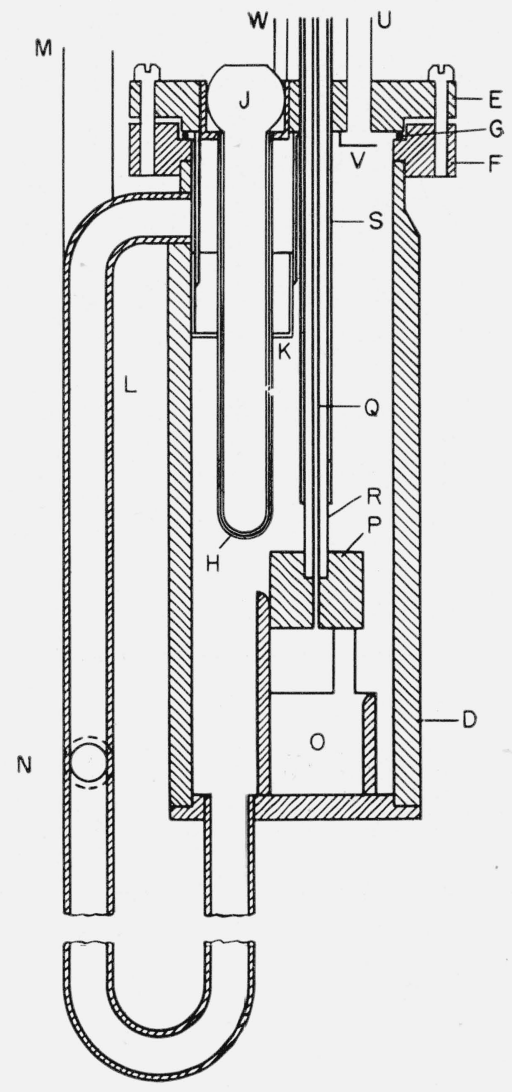

Figure 1. Equilibrium vessel.

D, copper cylinder; E, lid; F, flange; G, gold gasket; $\mathrm{H}$, thermometer well; $\mathrm{J}$, platinum resistance thermometer; $\mathrm{K}$, basket; $\mathrm{L}$, vapor lift pump; $\mathrm{M}$, vapor outlet capillary tube; R, driving tube; S, guide tube; U, vapor outlet tube; $V$, baffle; W, manometer tube.

point in the side wall near the top of the cylinder. The pumping action of the circulating vapors also thoroughly stirs the liquid in the cylinder of the equilibrium vessel.

Because of the low pressures expected in the equilibrium vessel during many of the measurements, it was not expected that liquid could be effectively removed by suction from the outside. Instead, a force pump was incorporated into the equilibrium vessel to apply the necessary pressure to force liquid through a capillary tube to an external sample receiver. The force pump consists of a cup, $\mathrm{O}$, of about $1 / 2$-in. diameter in the bottom of the equilibrium vessel, and a close fitting piston, $\mathrm{P}$, which can be trust into the cup by operation from outside the cryostat. The liquid trapped in the cup at the time the piston enters it is forced through the small tube, Q, having a 1/2-mm. bore, into a mercury filled receiving bulb. During the experiments there was leakage of liquid around the piston, but samples obtained were more than adequate in volume for all analyses that were desired. From a cup volume of approximately 1.5 $\mathrm{cm}^{3}$, a volume of 500 or $600 \mathrm{~cm}^{3}$ of gas, at room temperature, and slightly more than atmospheric pressure, is delivered in perhaps 10 seconds. Figure 2 is a sketch showing the working parts of the sam- 


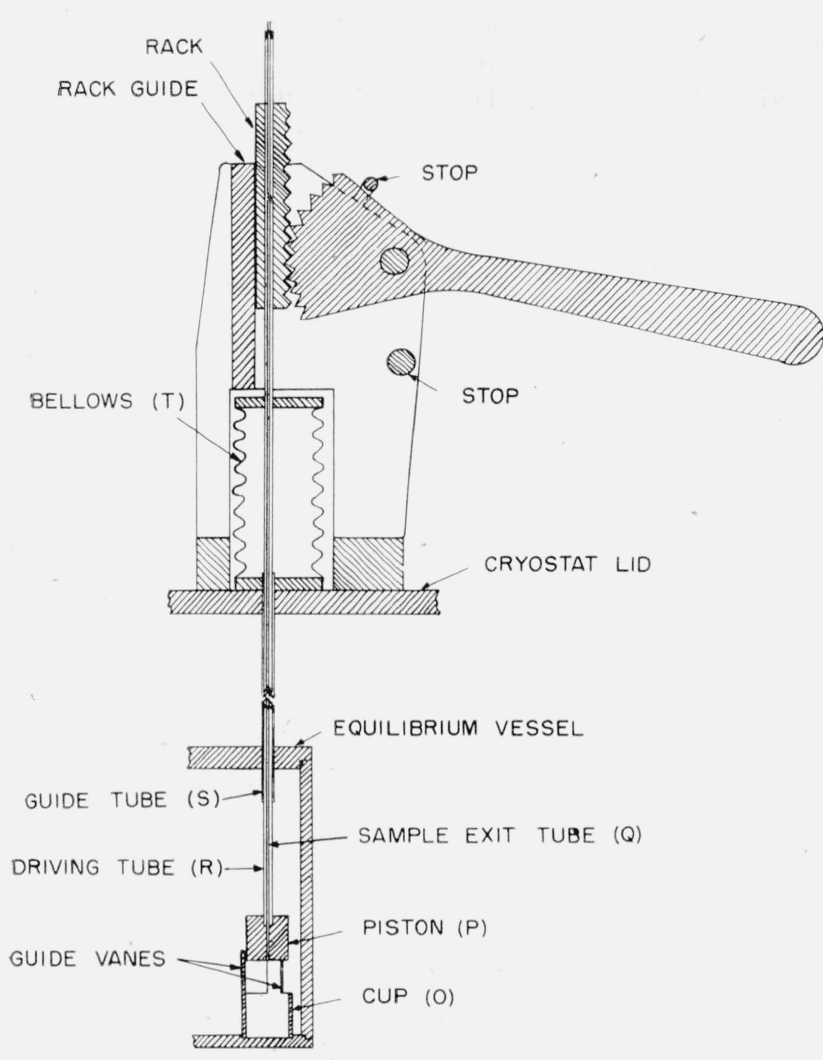

Figure 2. Liquid phase sampling device.

pling device. To force the piston into the cup, a thin wall $1 / 8$-in. monel tube, $R$, guided through the several enclosing chambers by a slightly larger thin wall monel tube, $\mathrm{S}$, is driven downward by a manually operated gear and rack. A sylphon bellows, $\mathrm{T}$, is used at the top of the cryostat to make a tight closure between the driving tube and the stationary guide tube.

The circulating gas leaves the equilibrium vessel through tube, U, which is protected from sprayed droplets by a baffle, $\mathrm{V}$, and during part of the experiments was covered with a pad of glass wool. A $1 / 16$-in. diameter tube, $W$, leads directly from the equilibrium vessel to the manometer. Electrical leads for the thermometer are brought into the evacuated chamber surrounding the equilibrium vessel via the vacuum pump line, a $1 / 2$-in. thin wall tube, not shown in the diagram, which projects into the evacuated space a short distance. The wires are wrapped several turns around this projecting end to insure their being at the isothermal bath temperature before leading to the thermometer.

\subsection{Gas Circulation System}

The vapor mixture of oxygen and nitrogen was circulated through the liquid, providing an opportunity for equilibrium to be established between the liquid and sufficient vapor for analysis, and at the same time stirring the liquid. The vapor was brought out of the cryostat during a portion of the cycle. It

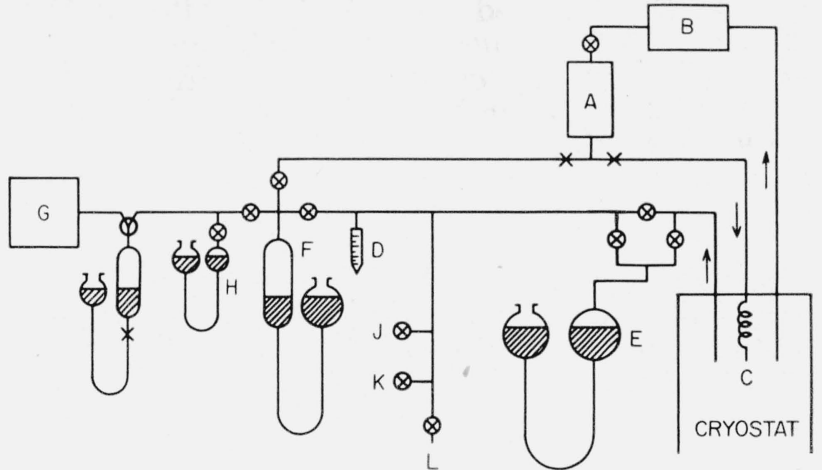

Figure 3. Gas handling system.

A, Vapor sample bulb; B, circulatory pump; C, tempering coil; D, graduated bulb; E, liquid sample receiving bulb; F, gas transfer bulb; G, analysis apparatus; H, auxiliary gas storage bulb; J, K, connections for oxygen and nitrogen supplies; L, connection for vacuum pump.

passed through a bulb, A (fig. 3), which in the $70^{\circ}$ and $77.5^{\circ}$ measurements had a volume of $500 \mathrm{~cm}^{3}$, but for the $65^{\circ}$ measurements was replaced by a bulb having a volume of $2,000 \mathrm{~cm}^{3}$, in order to provide sufficient vapor phase for analysis. The bulb could be closed off from the circulation line in order to remove a sample of the vapor for analysis. A circulatory pump, B, intended to cause movement of the gas without appreciably changing the volume of the system during any portion of its cycle, was used to force the gas mixture along the path from the gas reservoir bulb, into the equilibrium vessel via the vapor lift pump and return it to the gas reservoir bumb. At times a flowmeter, either an oil bubbler or a spherical ball, suspended in a small tube by the gas stream, was inserted in the line to monitor the flow. The circulatory pump used in these experiments was a commercially available flexible liner pump, in which a rotating eccentric cam presses the liner against a plastic enclosing block, and by its eccentric motion presses a bubble of gas forward, simultaneously admitting more gas behind the rotating part. By combination of motor shunts and different gear ratios a variety of circulation speeds was available.

When first operated with reduced pressure in the equilibrium system, the active flexible liner of the circulatory pump collapsed against the enclosing block, thus preventing any pumping action. It was found possible to rectify this situation by modifying the pump slightly, to permit a substantially equal vacuum to be placed external to the flexible liner. The circulating action of the pump could thus be maintained down to the lowest pressures encountered. The pump and the gas reservoir bulb were kept in a constant temperature oil bath to prevent changes of pressure due to changes in ambient temperature. This precaution was probably not necessary.

The gases were at room temperature entering the cryostat. It was found that if they were permitted to run directly to the equilibrium vessel a slow rise of the equilibrium vessel temperature occurred as long as circulation continued. A coil, C, of a few turns of copper tubing, was inserted in the flow line at the point where the gases entered the outermost 
liquid nitrogen bath. This coil tempered the gases sufficiently well to prevent rising temperatures in the equilibrium vessel due to transport of heat by the gases during circulation. This may be illustrated by the tests at the oxygen point on March 19 . The temperature of boiling oxygen was measured to be $89.82^{\circ}$ without circulation; with circulation, the temperature dropped to $89.77^{\circ}$. The temperature calculated from Hoge's vapor pressure data on oxygen was $0.0077^{\circ}$ higher than the observed temperature without circulation; it was $0.0025^{\circ}$ higher during circulation. After discontinuing the circulation, the calculated temperature remained $0.0007^{\circ}$ higher than the measured temperature. Similar results were obtained on another test March 26.

\subsection{Temperature and Pressure Measurement}

The thermometer used in the measurements was a platinum resistance thermometer of the capsule type, calibrated below the oxygen point against the provisional temperature scale established by Hoge and Brickwedde [6] at the Bureau.

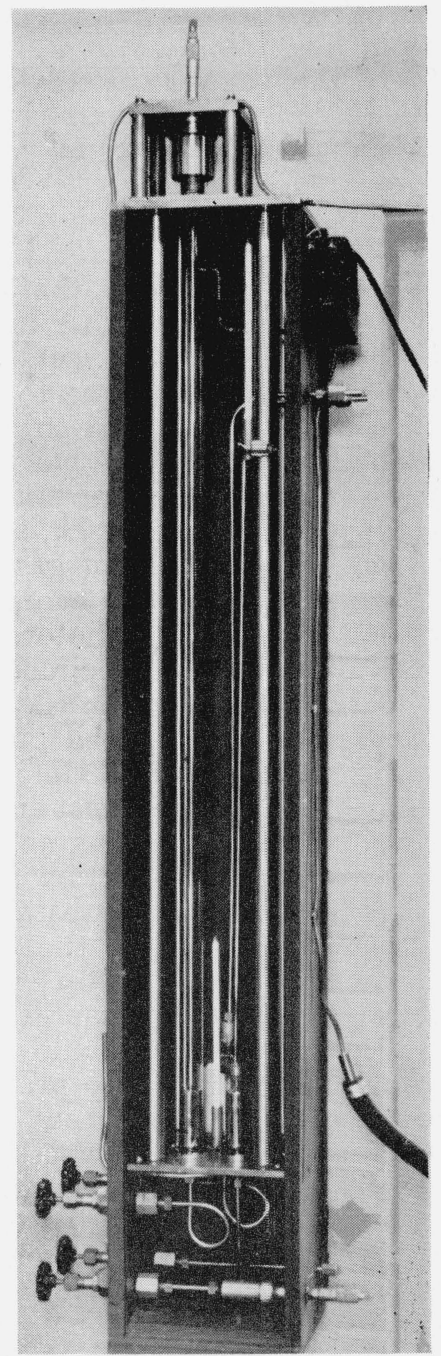

Figure 4. Precision manometer.
Pressure measurements were made with a precision manometer in which the positions of the mercury surfaces are located by a micrometer depth gage in conjunction with pointed stainless steel rods of calibrated lengths, and the contacts between the rods and the mercury are detected electrically. This manometer has been described [7]. Figure 4 is a picture of the manometer.

\subsection{Gas Handling and Analysis}

The material to be used in the equilibrium vessel was measured by first condensing to liquid in a graduated tube, D (fig. 3), of $15 \mathrm{~cm}^{3}$ capacity. The liquid was then transferred by distillation into the equilibrium vessel by way of the circulatory system. The circulatory pump was usually operated during: condensation. After equilibrium had been achieved in the apparatus, samples of gas from the liquid sample extractor were collected in a $1,000 \mathrm{~cm}^{3} \mathrm{bulb}$, $\mathrm{E}$, which was full of mercury before the sample was taken. After the sample was taken the gas was compressed to slightly greater than atmospheric pressure, to be retained until time for analysis. A $500-\mathrm{cm}^{3}$ bulb, F, which could be filled with mercury, was used for transferring gas from the storage bulb, $\mathrm{F}$, or the vapor sample bulb, $\mathrm{A}$, to the gas analysis apparatus, G. A third auxiliary bulb, H, of $200 \mathrm{~cm}^{3}$, was available to store a sample for later analysis if other bulbs were in use. The pure gases oxygen and nitrogen were introduced at $\mathrm{J}$ and $\mathrm{K}$. A vacuum pump, connected at $\mathrm{L}$ permitted evacuation of the whole gas handling system, or such parts of it as were necessary before transferring gases.

\subsection{Analysis of the Liquid_and Vapor Samples}

A commercial Shepard type volumetric gas analysis apparatus was used for analysis of the samples. Alkaline pyrogallol solutions for the absorption of oxygen were prepared according to the instructions of Kilday [8], to minimize carbon monoxide formation in the analysis of mixtures of high oxygen content. In general, two analyses were made of each sample, with the exceptions that in several of the early runs, three or more analyses were made in order to assure reproducibility, and in some of the runs, with solutions high in oxygen content at the lower temperatures, the vapor sample was insufficient for more than one analysis. For the 907 analyses performed on samples for which more than one analysis was made, the mean deviation was \pm 0.03 mole percent from the mean for the samples.

\subsection{Materials Used in the Experiments}

Commercially available compressed oxygen and nitrogen were used. The nitrogen was standard high purity dry nitrogen, purchased from the Linde Air Products Company. The oxygen was a special high purity grade, furnished through the courtesy of the Linde Air Products Company. Both were stated by the supplier to contain less than 0.005 percent of impurities. A study of the vapor pressure of the 
nitrogen has been previously reported [7]. The oxygen vapor pressure was checked at the normal boiling point, and the boiling point agreed to within $\pm 0.005^{\circ}$ with the value reported by Hoge [9].

\subsection{Experimental Procedure}

In preparation for an equilibrium measurement, the equilibrium vessel, previously cooled to the desired temperature, was filled with approximately $25 \mathrm{~cm}^{3}$ of oxygen and nitrogen, in proportions measured by condensing them separately in the graduated tube, D (fig. 3) before transfer to the equilibrium vessel. The gas handling system was then closed off from the circulation system. Circulation was maintained from 2 to $4 \mathrm{hr}$ at a rate generally about 275 to $300 \mathrm{~cm}^{3}$ of gas per minute except during certain experiments designed to determine whether variation of the circulating speed affected the measured equilibrium. This rate had been found in earlier tests of the vapor lift pump to be adequate to operate the pump. These tests had shown that a wide range of liquid levels in the equilibrium vessel and a wide range of vapor circulation rates would maintain action of the lift pump. The long periods of circulation were required by the slow approach of the equilibrium vessel temperature to a steady state.

After the temperature had remained constant to within a few hundredths of a degree for $15 \mathrm{~min}$ to $1 / 2 \mathrm{hr}$, a series of temperature and pressure measurements were made, at 2 -min intervals, either simultaneously by two observers or on alternate min by a single observer. At the conclusion of a third or fourth temperature reading the circulation was stopped, the vapor sample bulb closed off from the rest of the circulation system, and the liquid sample was taken. This total operation, including collection of the desired liquid sample, was completed generally in less than 1 min.

The procedure for collecting the liquid sample was adopted after several experiments, described in a discussion of the results. Equilibrium was established with the piston in the cup. The liquid sample flow line was opened to the vacuum pump. The piston was raised; one liquid sample was expelled into the vacuum system and rejected. Then the liquid sample flow line was opened to the sample collecting bulb, the piston raised again, and a second sample was forced out into the collecting bulb, where it was held for analysis. Analyses were generally performed during the same day the samples were collected, except that the last samples collected on one day were sometimes analyzed the next working day.

\section{Discussion of the Experimental Measurements}

The direct results of the experimental measurements are shown in tables 1 and 2 . Part $A$ is tbe $77.5^{\circ}$ isotherm, part $\mathrm{B}$ is the $70^{\circ}$ isotherm, and part $\mathrm{C}$ is the $65^{\circ}$ isotherm. Each experimental point is numbered, the numbers running consecutively through all experimental points in the chronological order in which the measurements were made. No points were discarded, although some might have been, for valid reasons which will be discussed later.

Of the experimental points, run number 32 is incomplete because the vapor sample was unintentionally discarded. The data observed are shown in columns 2 to 7 ; they are the absolute temperature, $\mathrm{T}$, the pressure in atmospheres, $\mathrm{P}$, the mole fractions in the liquid, $x_{\mathrm{N}_{2}}$ and $x_{\mathrm{O}_{2}}$, and the mole fractions in the vapor, $y_{\mathrm{N}_{2}}$ and $y_{\mathrm{O}_{2}}$. In column 1 is shown the date of the equilibrium measurement.

The treatment of the data is discussed in a later section; however, it will be helpful, for the present, to refer to figure 5, showing $\log _{e} \alpha$ plotted as functions of $x_{\mathrm{N}_{2}}$ for the three isotherms, in the discussion of the experiments which follows. In this figure the run number of each experimental point is shown to permit identification. The measurements represent a wide variety of detailed procedures used in bringing the liquid and vapor to equilibrium, and in sampling.

The first 10 runs on the $77.5^{\circ}$ isotherm represent attempts to learn the reproducibility of successive samples, to determine whether the sampling technique was adequate, and to examine the possibility of entrainment of liquid in the vapor. Run number 1 was made with the sampling piston lifted out of the cup, leaving in the cup an undisturbed volume of liquid which probably did not reach proper equilibrium, and which formed the bulk of the first sample. In this run oxygen was added first, and would be expected to form a disproportionate

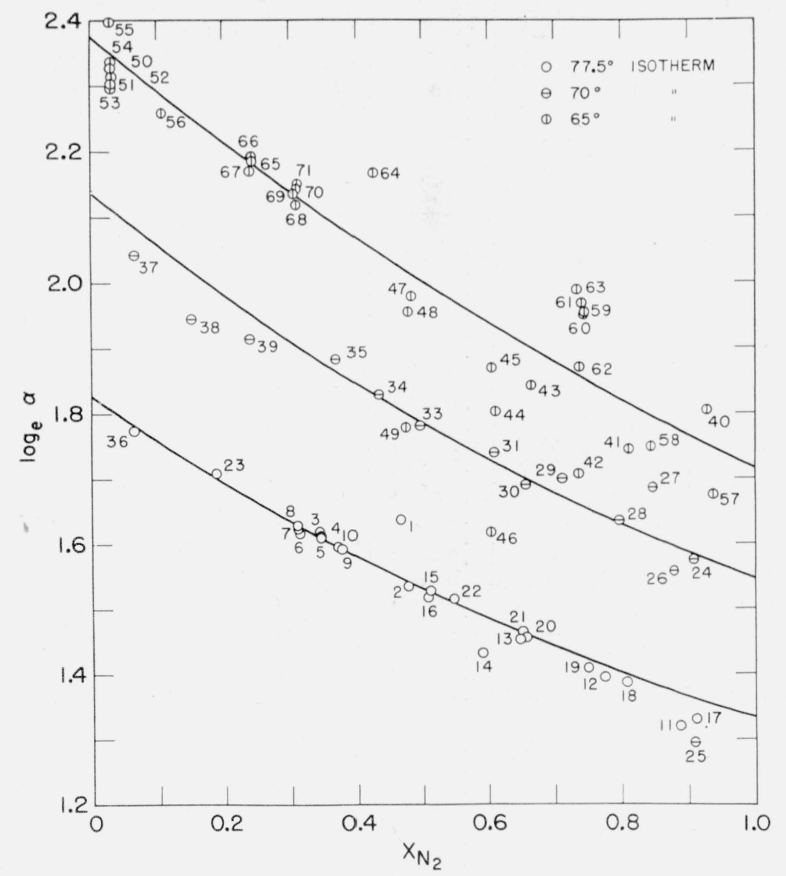

FIGURE 5. Logarithm of the separation coefficient for oxygennitrogen solutions.

The solid curves represent the separation coefficients calculated to be consistent with the linear activity coefficient functions:

$\frac{R T}{V_{1}} \log _{\odot} \gamma_{1}=A_{12} \phi_{2}^{2}$ in which $A_{12}$ in $\mathrm{cal} / \mathrm{cm}^{3}$ mole has the values 1.22 at $77.5^{\circ}, 1.38$ at $70^{\circ}$ and 1.47 at $65^{\circ} \mathrm{K}$. The experimental points are numbered in the order of their determination. $\bigcirc, 77.5^{\circ}$ Isotherm; $\Theta, 70^{\circ}$ Isotherm; $\odot, 65^{\circ}$ Isotherm. 
Table 1.

\begin{tabular}{|c|c|c|c|c|c|c|c|c|c|c|c|c|c|c|c|}
\hline Run & Date & $\begin{array}{c}T^{\circ} K \\
\text { (observed) }\end{array}$ & $P($ atm $)$ & $x_{\mathbf{N}_{2}}$ & $x_{\mathrm{O}_{2}}$ & $y_{\mathrm{N}_{2}}$ & $y_{\mathrm{O}_{2}}$ & $\alpha$ & $\begin{array}{l}\log _{e} \alpha \\
\text { (at } T \\
\text { obs) }\end{array}$ & $\begin{array}{c}P_{\mathrm{N}_{2}}^{\circ} \\
\text { (atm) } \\
(\text { at } T \\
\text { obs) }\end{array}$ & $\begin{array}{c}P_{\mathrm{O}_{2}}^{\circ} \\
\text { (atm) } \\
\text { (at } T \\
\text { obs) }\end{array}$ & $B_{\mathrm{N}_{2}}^{\prime}\left(P-P_{\mathrm{N}_{2}}^{\circ}\right)$ & $B_{\mathrm{O}_{2}}^{\prime}\left(P-P_{\mathrm{O}_{2}}^{\circ}\right)$ & $\begin{array}{l}\log _{e} a_{\mathrm{N}_{2}} \\
\text { (at } T \\
\text { obs) }\end{array}$ & $\begin{array}{c}\log _{e} a_{\mathrm{O}_{2}} \\
\text { (at } T \text { obs) }\end{array}$ \\
\hline \multicolumn{16}{|c|}{ Part A $\quad 77.5^{\circ}$ Isotherm } \\
\hline $\begin{array}{l}1 \\
2 \\
3 \\
4 \\
5\end{array}$ & $\begin{array}{l}\text { Apr. } 15 \\
\text { Apr. } 20\end{array}$ & $\begin{array}{l}77.9428 \\
77.8503 \\
77.7305 \\
77.7129 \\
77.6749\end{array}$ & $\begin{array}{r}0.67729 \\
.65792 \\
.53891 \\
.53755 \\
.53499\end{array}$ & $\begin{array}{r}0.4670 \\
.4789 \\
.3446 \\
.3461 \\
.3462\end{array}$ & $\begin{array}{r}0.5330 \\
.5211 \\
.6554 \\
.6539 \\
.6538\end{array}$ & $\begin{array}{r}0.8165 \\
8082 \\
.7248 \\
.7248 \\
.7248\end{array}$ & $\begin{array}{r}0.1835 \\
.1918 \\
.2752 \\
.2752 \\
.2752\end{array}$ & $\begin{array}{l}5.077 \\
4.586 \\
5.008 \\
4.975 \\
4.975\end{array}$ & $\begin{array}{l}1.624 \\
1.523 \\
1.611 \\
1.604 \\
1.604\end{array}$ & $\begin{array}{l}1.06987 \\
1.05851 \\
1.04382 \\
1.04168 \\
1.03707\end{array}$ & $\begin{array}{r}0.22232 \\
.21942 \\
.21562 \\
.21508 \\
.21389\end{array}$ & $\begin{array}{r}+0.0148 \\
.0152 \\
.0193 \\
.0193 \\
.0192\end{array}$ & $\begin{array}{r}-0.0221 \\
-.0213 \\
-.0158 \\
-.0157 \\
-.0157\end{array}$ & $\begin{array}{r}-0.6451 \\
-.6733 \\
-.9637 \\
-.9641 \\
-.9646\end{array}$ & $\begin{array}{r}-0.6037 \\
-.5745 \\
-.3900 \\
-.3899 \\
-.3892\end{array}$ \\
\hline $\begin{array}{r}6 \\
7 \\
8 \\
9 \\
10\end{array}$ & $\begin{array}{l}\text { Apr. } 21 \\
\text { Apr. } 23\end{array}$ & $\begin{array}{l}77.7450 \\
77.761 \\
77.765 \\
77.7256 \\
77.7925\end{array}$ & $\begin{array}{l}.50950 \\
.51022 \\
.51037 \\
.56364 \\
.56543\end{array}$ & $\begin{array}{l}.3128 \\
.3113 \\
.3103 \\
.3782 \\
.3735\end{array}$ & $\begin{array}{l}.6872 \\
.6887 \\
.6897 \\
.6218 \\
.6265\end{array}$ & $\begin{array}{l}.6945 \\
.6945 \\
.6945 \\
.7480 \\
.7444\end{array}$ & $\begin{array}{l}.3055 \\
.3055 \\
.3055 \\
.2520 \\
.2556\end{array}$ & $\begin{array}{l}\text { 4. } 993 \\
\text { 5. } 029 \\
\text { 5. } 053 \\
\text { 4. } 880 \\
\text { 4. } 885\end{array}$ & $\begin{array}{l}1.608 \\
1.615 \\
1.620 \\
1.585 \\
1.586\end{array}$ & $\begin{array}{l}1.04558 \\
1.04753 \\
1.04800 \\
1.04322 \\
1.05139\end{array}$ & $\begin{array}{l}.21607 \\
.21657 \\
.21671 \\
.21547 \\
.21757\end{array}$ & $\begin{array}{l}.0205 \\
.0205 \\
.0205 \\
.0183 \\
.0186\end{array}$ & $\begin{array}{l}-.0143 \\
-.0143 \\
-.0143 \\
-.0170 \\
-.0170\end{array}$ & $\begin{array}{r}-1.0630 \\
-1.0634 \\
-1.0636 \\
-0.8877 \\
-.8968\end{array}$ & $\begin{array}{l}-.3423 \\
-.3432 \\
-.3435 \\
-.4337 \\
-.4260\end{array}$ \\
\hline $\begin{array}{l}11 \\
12 \\
13 \\
14 \\
15\end{array}$ & $\begin{array}{l}\text { Apr. } 27 \\
\\
\text { Apr. } 29 \\
\text { A pr. } 30\end{array}$ & $\begin{array}{l}77.4185 \\
77.3534 \\
77.5388 \\
77.2694 \\
77.6315\end{array}$ & $\begin{array}{l}.91829 \\
.82865 \\
.75479 \\
.68718 \\
.66096\end{array}$ & $\begin{array}{l}.8875 \\
.7738 \\
.6478 \\
.5906 \\
.5119\end{array}$ & $\begin{array}{l}.1125 \\
.2262 \\
.3522 \\
.4094 \\
.4881\end{array}$ & $\begin{array}{l}.9673 \\
.9327 \\
.8871 \\
.8572 \\
.8280\end{array}$ & $\begin{array}{l}.0327 \\
.0673 \\
.1129 \\
.1428 \\
.1720\end{array}$ & $\begin{array}{l}\text { 3. } 750 \\
\text { 4. } 050 \\
\text { 4. } 270 \\
\text { 4. } 160 \\
\text { 4. } 591\end{array}$ & $\begin{array}{l}\text { 1. } 322 \\
\text { 1. } 399 \\
1.452 \\
\text { 1. } 426 \\
1.524\end{array}$ & $\begin{array}{l}1.00642 \\
0.99875 \\
1.02072 \\
0.98891 \\
1.03184\end{array}$ & $\begin{array}{r}20606 \\
.20408 \\
.20970 \\
.20159 \\
.21257\end{array}$ & $\begin{array}{l}.0034 \\
.0067 \\
.0103 \\
.0118 \\
.0142\end{array}$ & $\begin{array}{l}-.0350 \\
-.0308 \\
-.0269 \\
-.0241 \\
-.0220\end{array}$ & $\begin{array}{l}-.1215 \\
-.2497 \\
-.4113 \\
-.5063 \\
-.6200\end{array}$ & $\begin{array}{r}-1.9610 \\
-1.3281 \\
-0.9274 \\
-.7441 \\
-.6478\end{array}$ \\
\hline $\begin{array}{l}16 \\
17 \\
18 \\
19 \\
20\end{array}$ & $\begin{array}{ll}\text { May } & 3 \\
\text { May } & 4 \\
\text { May } & 5\end{array}$ & $\begin{array}{l}77.6254 \\
77.5463 \\
77.7410 \\
77.5113 \\
77.5014\end{array}$ & $\begin{array}{l}.65199 \\
.95303 \\
.89492 \\
.82861 \\
.75813\end{array}$ & $\begin{array}{l}.5073 \\
.9104 \\
.8070 \\
.7499 \\
.6563\end{array}$ & $\begin{array}{l}.4927 \\
.0896 \\
.1930 \\
.2501 \\
.3437\end{array}$ & $\begin{array}{l}.8238 \\
.9746 \\
.9433 \\
.9246 \\
.8911\end{array}$ & $\begin{array}{l}.1762 \\
.0254 \\
.0567 \\
.0754 \\
.1089\end{array}$ & $\begin{array}{l}\text { 4. } 541 \\
\text { 3. } 778 \\
\text { 3. } 978 \\
\text { 4. } 090 \\
\text { 4. } 287\end{array}$ & $\begin{array}{l}\text { 1. } 513 \\
\text { 1. } 329 \\
\text { 1. } 381 \\
\text { 1. } 409 \\
\text { 1. } 456\end{array}$ & $\begin{array}{l}1.03112 \\
1.02161 \\
1.04511 \\
1.01743 \\
1.01626\end{array}$ & $\begin{array}{l}21237 \\
.20993 \\
.21596 \\
.20887 \\
.20858\end{array}$ & $\begin{array}{l}.0145 \\
.0026 \\
.0057 \\
.0073 \\
.0100\end{array}$ & $\begin{array}{l}-.0215 \\
-.0365 \\
-.0331 \\
-.0304 \\
-.0270\end{array}$ & $\begin{array}{l}-.6377 \\
-.0926 \\
-.2078 \\
-.2764 \\
-.3983\end{array}$ & $\begin{array}{r}-.6359 \\
-2.1967 \\
-1.4815 \\
-1.2373 \\
-0.9520\end{array}$ \\
\hline $\begin{array}{l}21 \\
22 \\
23 \\
36\end{array}$ & $\begin{array}{lr}\text { May } & 6 \\
\text { May } & 7 \\
\text { May } & 31\end{array}$ & $\begin{array}{l}77.4755 \\
77.5168 \\
77.4172 \\
77.5600\end{array}$ & $\begin{array}{l}.75271 \\
.67928 \\
.38793 \\
.27691\end{array}$ & $\begin{array}{l}.6511 \\
.5477 \\
.1875 \\
.0632\end{array}$ & $\begin{array}{l}.3489 \\
.4523 \\
.8125 \\
.9368\end{array}$ & $\begin{array}{l}.8899 \\
.8464 \\
.5610 \\
.2841\end{array}$ & $\begin{array}{l}.1101 \\
.1536 \\
.4390 \\
.7159\end{array}$ & $\begin{array}{l}\text { 4. } 331 \\
4.549 \\
5.538 \\
5.882\end{array}$ & $\begin{array}{l}1.466 \\
1.515 \\
1.712 \\
1.772\end{array}$ & $\begin{array}{l}1.02240 \\
1.01809 \\
1.00605 \\
1.02327\end{array}$ & $\begin{array}{l}20778 \\
.20903 \\
.20601 \\
.21037\end{array}$ & $\begin{array}{l}.0104 \\
.0131 \\
.0239 \\
.0287\end{array}$ & $\begin{array}{l}-.0268 \\
-.0231 \\
-.0090 \\
-.0033\end{array}$ & $\begin{array}{r}-.4034 \\
-.5583 \\
-1.5071 \\
-2.5368\end{array}$ & $\begin{array}{l}-.9459 \\
-.7179 \\
-.1994 \\
-.0627\end{array}$ \\
\hline \multicolumn{16}{|c|}{ Part B $\quad 70^{\circ}$ Isotherm } \\
\hline $\begin{array}{l}24 \\
25 \\
26 \\
27 \\
28 \\
29 \\
30\end{array}$ & $\begin{array}{ll}\text { May } & 14 \\
\text { May } & 17 \\
\text { May } & 24 \\
\text { May } & 25 \\
\text { May } & 26\end{array}$ & $\begin{array}{l}69.7383 \\
70.2718 \\
70.1258 \\
70.3268 \\
70.3058 \\
70.2062 \\
70.1427\end{array}$ & $\begin{array}{r}0.33732 \\
.36663 \\
.34989 \\
.35274 \\
.33499 \\
.30578 \\
.28628\end{array}$ & $\begin{array}{r}0.9074 \\
.9084 \\
.8774 \\
.8479 \\
.7967 \\
.7117 \\
.6560\end{array}$ & $\begin{array}{r}0.0926 \\
.0916 \\
.1226 \\
.1521 \\
.2033 \\
.2883 \\
.3440\end{array}$ & $\begin{array}{r}0.9795 \\
.9729 \\
.9713 \\
.9675 \\
.9521 \\
.9307 \\
.9114\end{array}$ & $\begin{array}{r}0.0205 \\
.0271 \\
.0287 \\
.0325 \\
.0479 \\
.0693 \\
.0886\end{array}$ & $\begin{array}{l}\text { 4. } 874 \\
\text { 3. } 619 \\
\text { 4. } 729 \\
\text { 5. } 339 \\
\text { 5. } 072 \\
\text { 5. } 441 \\
\text { 5. } 392\end{array}$ & $\begin{array}{l}1.584 \\
1.286 \\
1.554 \\
1.675 \\
1.624 \\
1.694 \\
1.685\end{array}$ & $\begin{array}{r}0.36591 \\
.39566 \\
.38733 \\
.39883 \\
.39763 \\
.39190 \\
.38829\end{array}$ & $\begin{array}{r}0.05864 \\
.06474 \\
.06298 \\
.06534 \\
.06504 \\
.06386 \\
.06313\end{array}$ & $\begin{array}{r}+0.0016 \\
.0015 \\
.0020 \\
.0025 \\
.0033 \\
.0046 \\
.0055\end{array}$ & $\begin{array}{r}-0.0189 \\
-.0200 \\
-.0191 \\
-.0189 \\
-.0178 \\
-.0160 \\
-.0148\end{array}$ & $\begin{array}{r}-0.1005 \\
-.1022 \\
-.1288 \\
-.1534 \\
-.2172 \\
-.3154 \\
-.3922\end{array}$ & $\begin{array}{l}-2.1566 \\
-1.8943 \\
-1.8551 \\
-1.7593 \\
-1.4174 \\
-1.1191 \\
-0.9267\end{array}$ \\
\hline $\begin{array}{l}31 \\
32 \\
33 \\
34 \\
35\end{array}$ & $\begin{array}{l}\text { May } 27 \\
\text { May } 28\end{array}$ & $\begin{array}{l}70.2323 \\
70.2590 \\
70.2631 \\
70.2310 \\
70.3027\end{array}$ & $\begin{array}{l}.27588 \\
.26103 \\
.24328 \\
.22291 \\
.20605\end{array}$ & $\begin{array}{l}.6090 \\
\text { Disc: } \\
.4961 \\
.4325 \\
.3694\end{array}$ & $\begin{array}{c}.3904 \\
\text { rded } \\
.5031 \\
.5675 \\
.6306\end{array}$ & $\begin{array}{l}.8982 \\
.8270 \\
.8528 \\
.8246 \\
.7921\end{array}$ & $\begin{array}{l}.1018 \\
.1730 \\
.1472 \\
.1754 \\
.2079\end{array}$ & $\begin{array}{l}5.648 \\
5.876 \\
6.171 \\
6.504\end{array}$ & $\begin{array}{l}1.731 \\
1.771 \\
1.820 \\
1.872\end{array}$ & $\begin{array}{l}.39339 \\
.39492 \\
.39514 \\
.39332 \\
.39743\end{array}$ & $\begin{array}{l}.06414 \\
.06460 \\
.06460 \\
.06414 \\
.06504\end{array}$ & $\begin{array}{l}.0063 \\
.0071 \\
.0081 \\
.0091 \\
.0102\end{array}$ & $\begin{array}{l}-.0140 \\
-.0130 \\
-.0118 \\
-.0105 \\
-.0093\end{array}$ & $\begin{array}{l}-.4559 \\
-.5969 \\
-.6362 \\
-.7516 \\
-.8798\end{array}$ & $\begin{array}{l}-.8400 \\
-.3710 \\
-.6017 \\
-.5056 \\
-.4269\end{array}$ \\
\hline $\begin{array}{l}37 \\
38 \\
39\end{array}$ & $\begin{array}{cc}\text { June } & 1 \\
\text { June } & 2\end{array}$ & $\begin{array}{l}69.9039 \\
70.0302 \\
69.9396\end{array}$ & $\begin{array}{l}.08695 \\
.12237 \\
.15130\end{array}$ & $\begin{array}{l}.0643 \\
.1509 \\
.2381\end{array}$ & $\begin{array}{l}.9357 \\
.8491 \\
.7619\end{array}$ & $\begin{array}{l}.3471 \\
.5510 \\
.6799\end{array}$ & $\begin{array}{l}.6529 \\
.4490 \\
.3201\end{array}$ & $\begin{array}{l}7.736 \\
6.904 \\
6.798\end{array}$ & $\begin{array}{l}2.046 \\
\text { 1. } 932 \\
1.917\end{array}$ & $\begin{array}{l}.37495 \\
.38196 \\
.37692\end{array}$ & $\begin{array}{l}.06042 \\
.06197 \\
.06084\end{array}$ & $\begin{array}{l}.0156 \\
.0140 \\
.0122\end{array}$ & $\begin{array}{l}-.0018 \\
-.0040 \\
-.0061\end{array}$ & $\begin{array}{l}-2.5040 \\
-1.7203 \\
-1.2863\end{array}$ & $\begin{array}{l}-.0641 \\
-.1243 \\
-.2341\end{array}$ \\
\hline \multicolumn{16}{|c|}{ Part C $\quad 65^{\circ}$ Isotherm } \\
\hline $\begin{array}{l}40 \\
41 \\
42 \\
43 \\
44 \\
45\end{array}$ & $\begin{array}{cc}\text { June } & 7 \\
\text { June } & 8 \\
& \\
\text { June } & 9\end{array}$ & $\begin{array}{l}65.0858 \\
64.9475 \\
65.0300 \\
65.1480 \\
65.1997 \\
65.1115\end{array}$ & $\begin{array}{r}0.16359 \\
.14371 \\
.13533 \\
.12916 \\
.13079 \\
.12266\end{array}$ & $\begin{array}{r}0.9282 \\
.8112 \\
.7357 \\
.6644 \\
.6111 \\
.6057\end{array}$ & $\begin{array}{r}0.0718 \\
.1888 \\
.2643 \\
.3356 \\
.3889 \\
.3943\end{array}$ & $\begin{array}{r}0.9874 \\
.9610 \\
.9388 \\
.9255 \\
.9044 \\
.9084\end{array}$ & $\begin{array}{r}0.0126 \\
.0390 \\
.0612 \\
.0745 \\
.0956 \\
.0916\end{array}$ & $\begin{array}{l}6.063 \\
5.736 \\
5.509 \\
6.275 \\
6.021 \\
6.457\end{array}$ & $\begin{array}{l}1.802 \\
1.747 \\
1.706 \\
1.837 \\
1.795 \\
1.865\end{array}$ & $\begin{array}{r}0.17420 \\
.17010 \\
.17009 \\
.17608 \\
.17764 \\
.17497\end{array}$ & $\begin{array}{r}0.02334 \\
.02266 \\
.02308 \\
.02367 \\
.02395 \\
.02345\end{array}$ & $\begin{array}{r}+0.0606 \\
.0017 \\
.0022 \\
.0030 \\
.0030 \\
.0034\end{array}$ & $\begin{array}{r}-0.0117 \\
-.0102 \\
-.0094 \\
-.0088 \\
-.0089 \\
-.0083\end{array}$ & $\begin{array}{r}-0.0750 \\
-.2066 \\
-.3038 \\
-.3843 \\
-.4553 \\
-.4479\end{array}$ & $\begin{array}{r}-2.4388 \\
-1.4070 \\
-1.0341 \\
-0.9089 \\
-.7102 \\
-.7441\end{array}$ \\
\hline $\begin{array}{l}46 \\
47 \\
48 \\
49 \\
50\end{array}$ & $\begin{array}{cc}\text { June } 10 \\
\text { June } 11 \\
\text { June } 15\end{array}$ & $\begin{array}{l}65.0595 \\
65.2654 \\
65.1311 \\
65.2347 \\
64.8358\end{array}$ & $\begin{array}{l}.12261 \\
.10686 \\
.10433 \\
.10554 \\
.02876\end{array}$ & $\begin{array}{l}.6041 \\
.4834 \\
.4789 \\
.4748 \\
.0312\end{array}$ & $\begin{array}{r}.3959 \\
.5166 \\
.5211 \\
.5252 \\
.9688\end{array}$ & $\begin{array}{l}.8848 \\
.8702 \\
.8659 \\
.8413 \\
.2496\end{array}$ & $\begin{array}{l}.1152 \\
.1298 \\
.1341 \\
.1587 \\
.7504\end{array}$ & $\begin{array}{r}5.034 \\
7.163 \\
7.027 \\
5.864 \\
10.327\end{array}$ & $\begin{array}{l}1.616 \\
1.969 \\
1.950 \\
1.769 \\
2.335\end{array}$ & $\begin{array}{l}.17341 \\
.17966 \\
.17557 \\
.17872 \\
.16683\end{array}$ & $\begin{array}{l}.02324 \\
.02428 \\
.02357 \\
.02411 \\
.02214\end{array}$ & $\begin{array}{l}.0033 \\
.0047 \\
.0046 \\
.0047 \\
.0090\end{array}$ & $\begin{array}{l}-.0083 \\
-.0069 \\
-.0067 \\
-.0068 \\
-.0006\end{array}$ & $\begin{array}{r}-.4658 \\
-.6539 \\
-.6599 \\
-.6948 \\
-3.1370\end{array}$ & $\begin{array}{l}-.5062 \\
-.5667 \\
-.5279 \\
-.3710 \\
-.0263\end{array}$ \\
\hline $\begin{array}{l}51 \\
52 \\
53 \\
54 \\
55\end{array}$ & $\begin{array}{l}\text { June } 16 \\
\text { June } 17\end{array}$ & $\begin{array}{l}64.8623 \\
65.2375 \\
65.1427 \\
65.2942 \\
65.4351\end{array}$ & $\begin{array}{l}.02876 \\
.03086 \\
.03013 \\
.02978 \\
.03218\end{array}$ & $\begin{array}{l}.0310 \\
.0323 \\
.0312 \\
.0309 \\
.0299\end{array}$ & $\begin{array}{l}.9690 \\
.9677 \\
.9688 \\
.9691 \\
.9701\end{array}$ & $\begin{array}{l}.2437 \\
.2505 \\
.2412 \\
.2452 \\
.2489\end{array}$ & $\begin{array}{l}.7563 \\
.7495 \\
.7588 \\
.7548 \\
.7511\end{array}$ & $\begin{array}{r}10.071 \\
10.012 \\
9.871 \\
10.187 \\
10.751\end{array}$ & $\begin{array}{l}2.310 \\
2.304 \\
2.290 \\
2.321 \\
2.375\end{array}$ & $\begin{array}{l}.16761 \\
.17880 \\
.17592 \\
.18055 \\
.18493\end{array}$ & $\begin{array}{l}.02224 \\
.02411 \\
.02362 \\
.02439 \\
.02513\end{array}$ & $\begin{array}{l}.0090 \\
.0095 \\
.0094 \\
.0096 \\
.0097\end{array}$ & $\begin{array}{l}-.0006 \\
-.0006 \\
-.0005 \\
-.0004 \\
-.0006\end{array}$ & $\begin{array}{l}-3.1654 \\
-3.1319 \\
-3.1772 \\
-3.1551 \\
-3.1294\end{array}$ & $\begin{array}{l}-.0229 \\
-.0423 \\
-.0328 \\
-.0389 \\
-.0393\end{array}$ \\
\hline $\begin{array}{l}56 \\
57 \\
58 \\
59 \\
60\end{array}$ & $\begin{array}{cc}\text { June } & 18 \\
\text { June } & 22 \\
& \\
\text { June } & 23\end{array}$ & $\begin{array}{l}65.3413 \\
65.2718 \\
65.2116 \\
65.0456 \\
65.1846\end{array}$ & $\begin{array}{l}.04741 \\
.17028 \\
.15558 \\
.13793 \\
.14108\end{array}$ & $\begin{array}{l}.1073 \\
.9368 \\
.8452 \\
.7447 \\
.7442\end{array}$ & $\begin{array}{l}.8927 \\
.0632 \\
.1548 \\
.2553 \\
.2558\end{array}$ & $\begin{array}{l}.5311 \\
.9874 \\
.9689 \\
.9536 \\
.9531\end{array}$ & $\begin{array}{l}.4689 \\
.0126 \\
.0311 \\
.0464 \\
.0469\end{array}$ & $\begin{array}{l}9.423 \\
5.286 \\
5.704 \\
7.050 \\
6.989\end{array}$ & $\begin{array}{l}2.243 \\
1.665 \\
1.741 \\
1.953 \\
1.944\end{array}$ & $\begin{array}{l}.18201 \\
.17986 \\
.17801 \\
.17300 \\
.17718\end{array}$ & $\begin{array}{l}.02467 \\
.02428 \\
.02400 \\
.02313 \\
.02383\end{array}$ & $\begin{array}{l}.0086 \\
.0006 \\
.0014 \\
.0023 \\
.0023\end{array}$ & $\begin{array}{l}-.0019 \\
-.0121 \\
-.0109 \\
-.0096 \\
-.0098\end{array}$ & $\begin{array}{r}-1.9695 \\
-0.0668 \\
-.1648 \\
-.2718 \\
-.2737\end{array}$ & $\begin{array}{r}-.1062 \\
-2.4382 \\
-1.6123 \\
-1.2945 \\
-1.2914\end{array}$ \\
\hline $\begin{array}{l}61 \\
62 \\
63 \\
64 \\
65\end{array}$ & $\begin{array}{l}\text { June } 24 \\
\text { June } 25 \\
\text { June } 28\end{array}$ & $\begin{array}{l}65.2281 \\
64.9488 \\
65.0762 \\
65.3343 \\
64.9460\end{array}$ & $\begin{array}{l}.14168 \\
.13457 \\
.13675 \\
.10025 \\
.06712\end{array}$ & $\begin{array}{l}.7412 \\
.7365 \\
.7334 \\
.4252 \\
.2431\end{array}$ & $\begin{array}{l}.2588 \\
.2635 \\
.2666 \\
.5748 \\
.7569\end{array}$ & $\begin{array}{l}.9531 \\
.9482 \\
.9525 \\
.8644 \\
.7411\end{array}$ & $\begin{array}{l}.0469 \\
.0518 \\
.0475 \\
.1356 \\
.2589\end{array}$ & $\begin{array}{l}7.097 \\
6.508 \\
7.289 \\
8.618 \\
8.913\end{array}$ & $\begin{array}{l}1.960 \\
1.873 \\
1.986 \\
2.154 \\
2.188\end{array}$ & $\begin{array}{l}.17851 \\
.17013 \\
.17392 \\
.18179 \\
.17005\end{array}$ & $\begin{array}{l}.02405 \\
.02266 \\
.02329 \\
.02462 \\
.02266\end{array}$ & $\begin{array}{l}.0024 \\
.0022 \\
.0024 \\
.0052 \\
.0067\end{array}$ & $\begin{array}{l}-.0098 \\
-.0094 \\
-.0095 \\
-.0063 \\
-.0037\end{array}$ & $\begin{array}{r}-.2767 \\
-.2855 \\
-.2867 \\
-. .7358 \\
-1.2225\end{array}$ & $\begin{array}{r}-1.2963 \\
-1.1881 \\
-1.2865 \\
-0.6000 \\
-.2690\end{array}$ \\
\hline $\begin{array}{l}66 \\
67 \\
68 \\
69 \\
70 \\
71\end{array}$ & June 30 & $\begin{array}{l}65.2510 \\
65.1719 \\
65.2816 \\
65.2642 \\
65.3517 \\
65.4031\end{array}$ & $\begin{array}{l}.07013 \\
.06866 \\
.08124 \\
.08055 \\
.08225 \\
.08345\end{array}$ & $\begin{array}{l}.2424 \\
.2396 \\
.3092 \\
.3057 \\
.3098 \\
.3113\end{array}$ & $\begin{array}{l}.7576 \\
.7604 \\
.6908 \\
.6943 \\
.6902 \\
.6887\end{array}$ & $\begin{array}{l}.7389 \\
.7324 \\
.7862 \\
.7865 \\
.7899 \\
.7925\end{array}$ & $\begin{array}{l}.2611 \\
.2676 \\
.2138 \\
.2135 \\
.2101 \\
.2075\end{array}$ & $\begin{array}{l}8.845 \\
8.687 \\
8.216 \\
8.367 \\
8.377 \\
8.450\end{array}$ & $\begin{array}{l}2.180 \\
2.162 \\
2.106 \\
2.124 \\
2.126 \\
2.134\end{array}$ & $\begin{array}{l}.17921 \\
.17680 \\
.18016 \\
.17962 \\
.18230 \\
.18393\end{array}$ & $\begin{array}{l}.02417 \\
.02378 \\
.02433 \\
.02428 \\
.02472 \\
.02496\end{array}$ & $\begin{array}{l}.0070 \\
.0069 \\
.0063 \\
.0063 \\
.0064 \\
.0064\end{array}$ & $\begin{array}{r}-.0038 \\
-.0037 \\
-.0047 \\
-.0047 \\
-.0048 \\
-.0048\end{array}$ & $\begin{array}{l}-1.2337 \\
-1.2504 \\
-1.0307 \\
-1.0358 \\
-1.0256 \\
-1.0165\end{array}$ & $\begin{array}{l}-.2811 \\
-.2616 \\
-.3419 \\
-.3494 \\
-.3632 \\
-.3703\end{array}$ \\
\hline
\end{tabular}


Table 2.

\begin{tabular}{|c|c|c|c|c|c|c|c|c|c|c|c|c|c|c|c|}
\hline Run & $\log _{e} x_{\mathrm{N}_{2}}$ & $\log _{e} x \mathrm{O}_{2}$ & $\begin{array}{c}\log _{\bullet} \gamma_{\mathrm{N}_{2}} \\
\text { (at } T \\
\text { obs) }\end{array}$ & $\begin{array}{c}\log _{e} \gamma_{\mathrm{O}_{2}} \\
\text { (at } T \\
\text { obs) }\end{array}$ & $\Delta \mathrm{T}$ & $\Delta \log _{e} \gamma_{N_{2}}$ & $\Delta \log _{\bullet} \gamma_{\mathrm{O}_{2}}$ & $\Delta \log _{e} \alpha$ & $\begin{array}{c}\log _{e} \gamma_{\mathrm{N}_{2}} \\
\text { at } \\
\text { isotherm }\end{array}$ & $\begin{array}{c}\log _{e} \gamma_{\mathrm{O}_{2}} \\
\text { at } \\
\text { iso- } \\
\text { therm }\end{array}$ & $\begin{array}{l}\log _{\epsilon} \alpha \\
\text { at iso- } \\
\text { therm }\end{array}$ & $\frac{R T}{V_{\mathrm{N}_{2}}} \log _{e} \gamma_{\mathrm{N}_{2}}$ & $\frac{R T}{V_{\mathrm{O}_{2}}} \log _{e} \gamma_{\mathrm{O}_{2}}$ & $\phi_{\mathrm{N}_{2}}^{2}$ & $\phi_{\mathrm{O}_{2}}^{2}$ \\
\hline \multicolumn{16}{|c|}{ Part A $77.5^{\circ}$ Isotherm } \\
\hline $\begin{array}{l}1 \\
2 \\
3 \\
4 \\
5\end{array}$ & $\begin{array}{r}-0.7614 \\
-.7362 \\
-1.0654 \\
-1.0610 \\
-1.0607\end{array}$ & $\begin{array}{r}-0.6292 \\
-.6518 \\
-.4225 \\
-.4248 \\
-.4249\end{array}$ & $\begin{array}{r}0.1163 \\
.0629 \\
.1017 \\
.0969 \\
.0961\end{array}$ & $\begin{array}{l}0.0255 \\
.0773 \\
.0325 \\
.0349 \\
.0357\end{array}$ & $\begin{array}{r}-0.4428 \\
-.3808 \\
-.2305 \\
-.2129 \\
-.1749\end{array}$ & $\begin{array}{r}+0.0006 \\
.0005 \\
.0005 \\
.0005 \\
.0004\end{array}$ & $\begin{array}{r}0.0006 \\
.0006 \\
.0002 \\
.0002 \\
.0002\end{array}$ & $\begin{array}{l}0.013 \\
.011 \\
.007 \\
.007 \\
.005\end{array}$ & $\begin{array}{r}0.1169 \\
.0634 \\
.1022 \\
.0974 \\
.0965\end{array}$ & $\begin{array}{r}0.0261 \\
.0779 \\
.0327 \\
.0351 \\
.0359\end{array}$ & $\begin{array}{l}\text { 1. } 637 \\
\text { 1. } 534 \\
\text { 1. } 618 \\
\text { 1. } 611 \\
\text { 1. } 609\end{array}$ & $\begin{array}{r}0.518 \\
.281 \\
.453 \\
.432 \\
.428\end{array}$ & $\begin{array}{l}0.151 \\
.451 \\
.189 \\
.203 \\
.208\end{array}$ & $\begin{array}{r}0.2848 \\
.2977 \\
.1658 \\
.1672 \\
.1673\end{array}$ & $\begin{array}{r}0.2174 \\
.2065 \\
.3514 \\
.3494 \\
.3493\end{array}$ \\
\hline $\begin{array}{r}6 \\
7 \\
8 \\
9 \\
10\end{array}$ & $\begin{array}{r}-1.1622 \\
-1.1670 \\
-1.1702 \\
-0.9723 \\
-.9848\end{array}$ & $\begin{array}{l}-.3751 \\
-.3730 \\
-.3715 \\
-.4751 \\
-.4676\end{array}$ & $\begin{array}{l}.0992 \\
.1036 \\
.1066 \\
.0846 \\
.0880\end{array}$ & $\begin{array}{l}.0328 \\
.0298 \\
.0280 \\
.0414 \\
.0416\end{array}$ & $\begin{array}{l}-.2450 \\
-.261 \\
=.265 \\
-.2256 \\
-.2925\end{array}$ & $\begin{array}{l}.0006 \\
.0006 \\
.0007 \\
.0004 \\
.0006\end{array}$ & $\begin{array}{l}.0002 \\
.0002 \\
.0002 \\
.0002 \\
.0003\end{array}$ & $\begin{array}{l}.008 \\
.008 \\
.008 \\
.007 \\
.009\end{array}$ & $\begin{array}{l}.0998 \\
.1042 \\
.1073 \\
.0850 \\
.0886\end{array}$ & $\begin{array}{l}.0330 \\
.0300 \\
.0282 \\
.0416 \\
.0419\end{array}$ & $\begin{array}{l}1.616 \\
1.623 \\
\text { 1. } 628 \\
1.592 \\
1.595\end{array}$ & $\begin{array}{l}.443 \\
.462 \\
.476 \\
.377 \\
.393\end{array}$ & $\begin{array}{l}.191 \\
.174 \\
.163 \\
.241 \\
.243\end{array}$ & $\begin{array}{l}.1391 \\
.1378 \\
.1370 \\
.1961 \\
.1918\end{array}$ & $\begin{array}{l}.3933 \\
.3954 \\
.3968 \\
.3105 \\
.3160\end{array}$ \\
\hline $\begin{array}{l}11 \\
12 \\
13 \\
14 \\
15\end{array}$ & $\begin{array}{l}-.1194 \\
-.2564 \\
-.4342 \\
-.5266 \\
-.6696\end{array}$ & $\begin{array}{r}-2.1848 \\
-1.4863 \\
-1.0436 \\
-0.8931 \\
-.7172\end{array}$ & $\begin{array}{r}-.0021 \\
+.0067 \\
.0229 \\
.0203 \\
.0496\end{array}$ & $\begin{array}{l}.2238 \\
.1588 \\
.1162 \\
.1490 \\
.0694\end{array}$ & $\begin{array}{r}+.0815 \\
.1466 \\
-.0388 \\
+.2306 \\
-.1315\end{array}$ & $\begin{array}{r}.0000 \\
.0000 \\
.0000 \\
-.0002 \\
+.0001\end{array}$ & $\begin{array}{l}-.0003 \\
-.0005 \\
+.0001 \\
-.0005 \\
+.0002\end{array}$ & $\begin{array}{r}-.002 \\
-.004 \\
+.001 \\
.006 \\
.004\end{array}$ & $\begin{array}{r}-.0021 \\
.0067 \\
.0229 \\
.0201 \\
.0497\end{array}$ & $\begin{array}{l}.2235 \\
.1583 \\
.1163 \\
.1485 \\
.0696\end{array}$ & $\begin{array}{l}\text { 1. } 320 \\
\text { 1. } 395 \\
\text { 1. } 453 \\
\text { 1. } 432 \\
\text { 1. } 528\end{array}$ & $\begin{array}{r}-.009 \\
+.030 \\
.102 \\
.089 \\
.220\end{array}$ & $\begin{array}{r}1.295 \\
0.917 \\
.674 \\
.860 \\
.403\end{array}$ & $\begin{array}{l}.8306 \\
.6680 \\
.4986 \\
.4271 \\
.3342\end{array}$ & $\begin{array}{l}.0078 \\
.0334 \\
.0864 \\
.1201 \\
.1780\end{array}$ \\
\hline $\begin{array}{l}16 \\
17 \\
18 \\
19 \\
20\end{array}$ & $\begin{array}{l}-.6786 \\
-.0939 \\
-.2144 \\
-.2878 \\
-.4211\end{array}$ & $\begin{array}{r}-.7078 \\
-2.4129 \\
-1.6451 \\
-1.3859 \\
-1.0680\end{array}$ & $\begin{array}{l}.0409 \\
.0013 \\
.0066 \\
.0114 \\
.0228\end{array}$ & $\begin{array}{l}.0719 \\
.2162 \\
.1636 \\
.1486 \\
.1160\end{array}$ & $\begin{array}{l}-.1254 \\
-.0463 \\
-.2410 \\
-.0113 \\
-.0014\end{array}$ & $\begin{array}{l}.0001 \\
.0000 \\
.0000 \\
.0000 \\
.0000\end{array}$ & $\begin{array}{l}.0002 \\
.0002 \\
.0009 \\
.0000 \\
.0000\end{array}$ & $\begin{array}{l}.004 \\
.001 \\
.006 \\
.000 \\
.000\end{array}$ & $\begin{array}{l}.0410 \\
.0013 \\
.0066 \\
.0114 \\
.0228\end{array}$ & $\begin{array}{l}.0721 \\
.2164 \\
.1645 \\
.1486 \\
.1160\end{array}$ & $\begin{array}{l}1.517 \\
\text { 1. } 330 \\
1.387 \\
1.409 \\
1.456\end{array}$ & $\begin{array}{l}.182 \\
.006 \\
.029 \\
.051 \\
.101\end{array}$ & $\begin{array}{r}.418 \\
1.254 \\
0.953 \\
.861 \\
.672\end{array}$ & $\begin{array}{l}.3289 \\
.8643 \\
.7145 \\
.6346 \\
.5094\end{array}$ & $\begin{array}{l}.1819 \\
.0049 \\
.0239 \\
.0414 \\
.0820\end{array}$ \\
\hline $\begin{array}{l}21 \\
22 \\
23\end{array}$ & $\begin{array}{r}-.4291 \\
-.6020 \\
-1.6740\end{array}$ & $\begin{array}{r}-1.0530 \\
-0.7934 \\
-.2076\end{array}$ & $\begin{array}{l}.0257 \\
.0437 \\
.1669\end{array}$ & $\begin{array}{l}.1071 \\
.0755 \\
.0082\end{array}$ & $\begin{array}{l}+.0245 \\
-.0168 \\
+.0828\end{array}$ & $\begin{array}{r}.0000 \\
.0000 \\
-.0003 \\
\end{array}$ & $\begin{array}{r}-.0001 \\
.0000 \\
.0000\end{array}$ & $\begin{array}{r}-.001 \\
-.000 \\
-.003\end{array}$ & $\begin{array}{l}.0257 \\
.0437 \\
.1666\end{array}$ & $\begin{array}{l}.1070 \\
.0755 \\
.0082\end{array}$ & $\begin{array}{l}\text { 1. } 465 \\
\text { 1. } 515 \\
\text { 1. } 709 \\
1.774\end{array}$ & $\begin{array}{l}.114 \\
.194 \\
.739\end{array}$ & $\begin{array}{l}.620 \\
.437 \\
.048\end{array}$ & $\begin{array}{l}.5027 \\
.3755 \\
.0537\end{array}$ & $\begin{array}{l}.0847 \\
.1499 \\
.5903 \\
.8452\end{array}$ \\
\hline 36 & -2.7615 & -.0653 & .2247 & .0026 & & +.0003 & .0000 & +.002 & .2250 & .0026 & 1. 774 & .998 & & & .8453 \\
\hline \multicolumn{16}{|c|}{ Part B $70^{\circ}$ Isotherm } \\
\hline $\begin{array}{l}24 \\
25 \\
26 \\
27 \\
28 \\
29 \\
30\end{array}$ & $\begin{array}{r}-0.0971 \\
-.0961 \\
-.1308 \\
-.1650 \\
-.2273 \\
-.3401 \\
-.4216\end{array}$ & $\begin{array}{l}-2.3794 \\
-2.3903 \\
-2.0988 \\
-1.8832 \\
-1.5931 \\
-1.2437 \\
-1.0671\end{array}$ & $\begin{array}{r}-0.0034 \\
-.0061 \\
+.0020 \\
.0116 \\
.0101 \\
.0247 \\
.0294\end{array}$ & $\begin{array}{r}0.2228 \\
.4960 \\
.2437 \\
.1239 \\
.1757 \\
.1246 \\
.1404\end{array}$ & $\begin{array}{r}+0.2617 \\
-.2718 \\
-.1258 \\
-.3268 \\
-.3058 \\
-.2062 \\
-.1427\end{array}$ & $\begin{array}{r}+0.0000 \\
.0000 \\
.0000 \\
.0000 \\
.0001 \\
.0001 \\
.0001\end{array}$ & $\begin{array}{r}-0.0012 \\
+.0012 \\
.0005 \\
.0013 \\
.0011 \\
.0006 \\
.0004\end{array}$ & $\begin{array}{r}-0.009 \\
+.009 \\
.004 \\
.011 \\
.011 \\
.007 \\
.005\end{array}$ & $\begin{array}{r}-0.0034 \\
-.0061 \\
+.0020 \\
.0116 \\
.0102 \\
.0248 \\
.0295\end{array}$ & $\begin{array}{r}0.2216 \\
.4972 \\
.2442 \\
.1252 \\
.1768 \\
.1252 \\
.1408\end{array}$ & $\begin{array}{l}\text { 1. } 575 \\
\text { 1. } 295 \\
\text { 1. } 558 \\
\text { 1. } 686 \\
\text { 1. } 635 \\
\text { 1. } 701 \\
\text { 1. } 690\end{array}$ & $\begin{array}{r}-0.014 \\
-.025 \\
+.008 \\
.048 \\
.042 \\
.103 \\
.123\end{array}$ & $\begin{array}{r}1.194 \\
2.678 \\
1.316 \\
0.674 \\
.952 \\
.674 \\
.758\end{array}$ & $\begin{array}{l}0.8591 \\
.8606 \\
.8145 \\
.7714 \\
.6977 \\
.5797 \\
.5062\end{array}$ & $\begin{array}{r}0.0053 \\
.0052 \\
.0095 \\
.0148 \\
.0271 \\
.0569 \\
.0832\end{array}$ \\
\hline $\begin{array}{l}31 \\
32\end{array}$ & -.4959 & -0.9406 & .0400 & .1006 & $\begin{array}{l}-.2323 \\
-.2590\end{array}$ & .0002 & .0005 & .009 & .0402 & 1011 & 1. 740 & .167 & .545 & .4465 & .1101 \\
\hline 33 & -7010 & -.6870 & .0648 & .0853 & -.2631 & .0003 & .0004 & .010 & .0651 & .0857 & 1. 781 & .271 & .462 & .3138 & .1934 \\
\hline $\begin{array}{l}34 \\
35\end{array}$ & $\begin{array}{l}-.8382 \\
-.9959\end{array}$ & $\begin{array}{l}-.5665 \\
-.4611\end{array}$ & $\begin{array}{l}.0866 \\
.1161\end{array}$ & $\begin{array}{l}.0609 \\
.0342\end{array}$ & $\begin{array}{l}-.2310 \\
-.3027\end{array}$ & $\begin{array}{l}.0004 \\
.0006\end{array}$ & .0003 & $\begin{array}{l}.009 \\
.012\end{array}$ & $\begin{array}{l}.0870 \\
1167\end{array}$ & $\begin{array}{l}.0612 \\
0345\end{array}$ & $\begin{array}{l}1.829 \\
1.884\end{array}$ & $\begin{array}{r}.362 \\
486\end{array}$ & .330 & .2466 & .2534 \\
\hline 37 & -2.7442 & -.0665 & .2402 & .0024 & $\begin{array}{r}.0961 \\
+\end{array}$ & -.0005 & .0000 & -.004 & 2397 & .0024 & 2. 042 & 998 & .013 & .0066 & 8438 \\
\hline 38 & -1.8911 & -.1636 & .1708 & .0393 & -.0302 & +.0001 & .0000 & +.013 & $\begin{array}{l}.1709 \\
.1798\end{array}$ & .0393 & $\begin{array}{l}1.945 \\
\text {. }\end{array}$ & .71 & .21 & $\begin{array}{r}.0000 \\
.0349\end{array}$ & .64015 \\
\hline 39 & -1.4351 & -.2719 & .1488 & .0378 & +.0604 & -.0002 & .0000 & -.003 & .1486 & .0378 & 1.914 & .619 & .204 & .0829 & .5070 \\
\hline \multicolumn{16}{|c|}{ Part C $65^{\circ}$ Isotherm } \\
\hline 40 & -0.0745 & -2.6339 & -0.0005 & 0.1951 & -0.0858 & +0.0000 & +0.0004 & +0.003 & -0.0005 & 0.1955 & 1.805 & -0.002 & 0.997 & 0.8896 & 0.0032 \\
\hline 41 & -.2092 & 1. 6671 & $\begin{array}{r}+.0026 \\
\end{array}$ & .2601 & $\begin{array}{l}+.0525 \\
\end{array}$ & .0000 & -.0002 & -.002 & $\begin{array}{r}+.0026 \\
\end{array}$ & .2599 & 1.745 & $\begin{array}{l}+.010 \\
\end{array}$ & 1. 326 & .7171 & .0235 \\
\hline $\begin{array}{l}11 \\
42\end{array}$ & -30 & 7 & .00 & .29 & -.0 & .00 & +.0001 & $\begin{array}{r}+.001 \\
+\end{array}$ & .0031 & .29 & & .01 & 1. & .6112 & .0476 \\
\hline 43 & $\begin{array}{l}-.40 \\
-.40\end{array}$ & 1. & .02 & .18 & -.1 & .00 & .0004 & .00 & .0253 & .18 & & .10 & 0. & .01157 & .0795 \\
\hline 44 & -.4924 & -0.94 & 0371 & .23 & -.19 & .000 & .0005 & & .03 & .23 & & .14 & & .4476 & .1096 \\
\hline 45 & -.5014 & -.9306 & .0535 & .1864 & -.1115 & .0001 & .0003 & .005 & .0536 & .1867 & 1.870 & .213 & 0.953 & .4409 & .1129 \\
\hline $\begin{array}{l}46 \\
47\end{array}$ & $\begin{array}{l}-.5040 \\
-.7269\end{array}$ & $\begin{array}{l}-.9266 \\
-.6605\end{array}$ & .0382 & .4204 & $\begin{array}{l}-.0595 \\
-.2654\end{array}$ & .0000 & .0001 & .002 & .0382 & .4205 & $\begin{array}{l}1.618 \\
1980\end{array}$ & .151 & & .4389 & $\begin{array}{r}1139 \\
2058\end{array}$ \\
\hline $\begin{array}{l}47 \\
48\end{array}$ & $\begin{array}{l}-.7269 \\
-.7363\end{array}$ & $\begin{array}{l}-.6605 \\
-6518\end{array}$ & & $\begin{array}{r}.0938 \\
1239\end{array}$ & $\begin{array}{l}-.2654 \\
-.1311\end{array}$ & $\begin{array}{r}.0003 \\
0002\end{array}$ & $\begin{array}{r}.0004 \\
0002\end{array}$ & .011 & $\begin{array}{l}.0733 \\
0766\end{array}$ & $\begin{array}{r}.0942 \\
.1241\end{array}$ & $\begin{array}{l}1.980 \\
1.956\end{array}$ & .291 & $\begin{array}{l}0.481 \\
633\end{array}$ & $\begin{array}{r}.2986 \\
2937\end{array}$ & $\begin{array}{l}.2058 \\
2099\end{array}$ \\
\hline $\begin{array}{l}48 \\
49\end{array}$ & -.7 & & & .273 & $\begin{array}{l}-.10 \\
-.23 \\
\end{array}$ & & .0003 & .01 & & .2733 & & & & .2896 & \\
\hline 50 & -3.4673 & -.0317 & .3303 & .0054 & +.1642 & $\begin{array}{r}-.0009 \\
-.009\end{array}$ & .0000 & -.008 & .3294 & .0054 & 2. 327 & $\begin{array}{l}.139 \\
1.306\end{array}$ & 0.028 & .0015 & 29 \\
\hline 51 & -3.4738 & -.0315 & .3084 & .0086 & .1377 & -.0008 & .0000 & -.007 & .3076 & +.0086 & 2. 303 & 1. 220 & .044 & .0015 & .9235 \\
\hline 52 & -3.4327 & & & & & +.0014 & & & & & & 1.19 & & .0017 & .9203 \\
\hline 53 & -3.4673 & -.0 & .29 & -.00 & -.14 & .00 & 0 & .007 & .2 & -.8 & & 1.153 & - . & .0015 & .9229 \\
\hline 54 & -3.4770 & -.0 & 3 & -.00 & -.2 & .00 & .00 & .015 & & -.00 & & 1. 28 & & .0015 & .9237 \\
\hline 55 & -3.5099 & -.0297 & .3805 & -.0096 & -.4351 & .0025 & .0000 & .022 & .3830 & -0096 & 2. 397 & 1.519 & -.049 & .0014 & .9262 \\
\hline 56 & -2.2321 & -.1134 & .2626 & +.0072 & -.3413 & .0016 & .0000 & .016 & .2642 & +.0072 & 2. 259 & 1. 048 & +.037 & .0179 & .7505 \\
\hline 57 & & & -.0015 & .3232 & -.27 & .00 & .001 & .01 & -.0015 & .3245 & & & & & .0025 \\
\hline 58 & $\begin{array}{l}-.1681 \\
\end{array}$ & & +.0033 & .25 & -.2 & .00 & .0 & .0 & +.0 & 2 & & $\begin{array}{l}0.013 \\
+.013\end{array}$ & 1. & & .0155 \\
\hline 59 & -.2948 & -1.3653 & .0230 & .0708 & -.04 & .0000 & .0001 & .002 & .0230 & .0709 & & .091 & 0.362 & .6234 & .0043 \\
\hline 60 & -.2954 & -1.3634 & .0217 & .0720 & -.1846 & .0001 & .0006 & .007 & .0218 & .0726 & 1. 951 & .086 & .370 & .6228 & .0444 \\
\hline 61 & -.2995 & -1.3517 & .0228 & .0554 & -.2281 & .0001 & .0007 & .009 & .0229 & .0561 & 1. 969 & .091 & .286 & .6187 & $.0455^{\circ}$ \\
\hline 62 & $\begin{array}{l}-.3059 \\
30101\end{array}$ & -1.3337 & .0204 & .1456 & +.0512 & .0000 & -.0002 & -.002 & .0204 & .1454 & 1. 871 & .081 & .742 & .6128 & .0472 \\
\hline $\begin{array}{l}63 \\
64\end{array}$ & $\begin{array}{l}-.3101 \\
-.8552\end{array}$ & $\begin{array}{l}-1.3220 \\
-0.5537\end{array}$ & $\begin{array}{l}.0234 \\
1194\end{array}$ & $\begin{array}{r}.0355 \\
-0463\end{array}$ & $\begin{array}{l}-.0762 \\
-.3343\end{array}$ & .0000 & $\begin{array}{l}+.0002 \\
.0004\end{array}$ & +.003 & .0234 & $\begin{array}{r}.0357 \\
-0459\end{array}$ & $\begin{array}{l}\text { 1. } 989 \\
2168\end{array}$ & .093 & .182 & .6081 & .0485 \\
\hline $\begin{array}{l}64 \\
65\end{array}$ & $\begin{array}{r}-.8552 \\
-1.4143\end{array}$ & $\begin{array}{r}-0.5537 \\
-.2785\end{array}$ & $\begin{array}{l}.1194 \\
.1918\end{array}$ & $\begin{array}{l}-.0463 \\
+.0095\end{array}$ & $\begin{array}{l}-.3343 \\
+.0540\end{array}$ & $\begin{array}{r}.0005 \\
-.0002\end{array}$ & $\begin{array}{l}.0004 \\
.0000\end{array}$ & $\begin{array}{r}.014 \\
-.002\end{array}$ & $\begin{array}{l}.1199 \\
.1916\end{array}$ & $\begin{array}{r}-.0459 \\
+.0095\end{array}$ & $\begin{array}{l}2.168 \\
2.186\end{array}$ & $\begin{array}{l}.475 \\
.760\end{array}$ & $\begin{array}{r}-.234 \\
+.048\end{array}$ & $\begin{array}{l}.2379 \\
.0855\end{array}$ & $\begin{array}{l}.2623 \\
.5007\end{array}$ \\
\hline 66 & -1.4172 & & & -.0035 & & +.0008 & & +.012 & 18 & -.0034 & & & & & \\
\hline 67 & & -.2739 & .1784 & +.0123 & -.1719 & & .0 & 8 & 1 & & & & $\begin{array}{r}.063 \\
+.06\end{array}$ & .0832 & .5064 \\
\hline 68 & -1.1738 & -.3699 & .1431 & .0280 & -.2 & .00 & .0 & .01 & 1 & .0282 & & .570 & .144 & .1334 & .4028 \\
\hline 69 & -1.1851 & -.364 & .1493 & .0154 & -.2 & .00 & & .012 & & .0156 & & . 595 & .080 & & 978 \\
\hline 70 & -1.1718 & -.3708 & .1462 & .0076 & -.3517 & .0009 & & .016 & & & & & .040 & & .4021 \\
\hline 71 & -1.1670 & -.3730 & .1505 & .0027 & -.4031 & .0010 & .0003 & .018 & .1515 & .0030 & 2. 152 & .601 & .015 & .1351 & .4001 \\
\hline
\end{tabular}


amount of the liquid sample. It is therefore justifiable to disregard this run as unsatisfactory in procedure. Run number 2, made after further circulation of the same liquid, gives a value of $\log _{e} \alpha$ in good agreement with neighboring points. Runs 3,4 , and 5 form a series in which, after circulating with the piston lowered into the cup, three liquid samples were taken within an hour from the same liquid mixture, and the vapor sample was taken only at the end. Here, again, oxygen was added first and nitrogen last. Runs 6, 7 , and 8 form a similar series, in which 1 vapor sample and 3 liquid samples were taken from one liquid mixture; but in this case oxygen was added last. There is an obvious tendency for the points in each series to approach a common line (when plotted as $\log _{e} \alpha$ ), and in each case the earliest run differs from later runs in the direction to be expected if the material in the liquid sample was disproportionately rich in the component added to the equilibrium vessel first. Thereupon the obvious course was adopted of discarding the first liquid sample completely, and was followed in all later runs.

The possibility of entrainment of liquid in the vapor stream was investigated in the next series of measurements, represented by runs 9 and 10 . The device used to detect entrainment was the variation of the circulation rate. Run 9 was made with the circulating pump operating at full speed until the samples were taken; while in run 10, after full speed circulation of the same mixture for three quarters of an hour, the pump was cut to approximately $1 / 4$ speed for $50 \mathrm{~min}$. The values of $\log _{e} \alpha$ obtained from runs 9 and 10 are in excellent agreement with each other and with the later measurements of all the preceding series. Therefore it was concluded at this point that a suitable sampling procedure had been found, and that the samples obtained were satisfactorily reproducible.

In carrying out the remainder of the measurements, it became apparent after a time that over-all reproducibility of a point was not as good as had been anticipated. Because of lingering doubts about the possibility of entrainment, a pad of glass wool to catch liquid droplets was placed over the vapor outlet tube as early as run 11, and remained there until run 50, at which time it was replaced by a metal baffle.

The data of the $77.5^{\circ}$ isotherm form a reasonably smooth pattern of points; the data of the $70^{\circ}$ isotherm are somewhat more scattered. The data of the $65^{\circ}$ isotherm have become seriously irregular. In the course of the measurements, numerous attempts were made to discover the cause of the irregularities and lack of reproducibility, and these will be touched on briefly.

a. No consistent effect on $\log _{e} \alpha$ could be traced to upward or downward temperature drift of the equilibrium vessel.

b. Repeated checks of the analyses showed that the scatter of the data could not be accounted for on the basis of differences in analysis of several portions of the same sample. c. No effect could be attributed to the storage of some samples overnight before analysis, or to the storage of a few of the early samples under reduced pressure. All samples made after run 23 were stored under pressure slightly greater than atmospheric until analyzed.

d. The thermal insulating vacuum space around the equilibrium vessel was usually filled with helium during changes of temperature, and was usually filled with helium overnight. Leakage of helium into the equilibrium vessel would result in high apparent values of nitrogen. The residue of an analysis of a sample, obtained after the equilibrium vessel had stood overnight surrounded by helium, was examined by mass spectrometer. No helium was detected.

e. It was suspected that the fullness of the equilibrium vessel might affect the efficiency of stirring by the circulation. Runs 50 through 55 and 59 through 63 were made without addition of new solution to the equilibrium vessel. In the latter series of runs the volume of solution diminished from $29 \mathrm{~cm}^{3}$ to $17 \mathrm{~cm}^{3}$. While there is no question that an effect was produced, and series 50 through 55 seems to follow a cyclic pattern, no basis has been arrived at for concluding that one value was better than another.

f. Examination of the data as a whole reveals two suggestive trends: that the scatter of the points becomes worse as the experiments continue, and that the scatter of points becomes worse the lower the temperature. Because the data were obtained in order from higher to lower temperature these two trends are impossible to assess separately.

The dependence of the scatter upon time would require a deterioration of the apparatus or an unnoticed change in the experimental procedure. Most parts of the apparatus were checked from time to time during the experiments without any indication of faulty operation. One possible source of difficulty, which was not specifically checked, is that the leaky joint between the thermometer well and the basket became clogged and failed to allow the mixture to drain through. This would have resulted in a pocket of unstirred liquid which might have contributed erratically to the composition of the mixture.

More likely, however, appears to be the effect of the much lower pressures prevailing at the lower temperatures. The lower pressures would mean that if entrainment of liquid droplets in the vapor stream occurred, a given size drop of liquid would disturb the vapor composition more than at higher pressures.

In another way, too, the lower mass of vapor present at the lower pressures could play a part. In the operation of the vapor lift pump a small pressure differential must build up between the inside and outside of the equilibrium vessel. Some condensation must occur on the high pressure side as a result, and some evaporation occurs at the lower pressure surface. If, at a low pressure, the condensation and evaporation should be sufficient to account for the 
total mass of gas flowing through the circulating system, stirring of the liquid would cease to be systematic. Although sporadic stirring might occur, the compositions measured would very probably be unreproducible. Evidence for the existence of this behavior was seen in the fluctuations of an auxiliary manometer connected to the flow system, which were obvious at pressures above $250 \mathrm{~mm}$ but diminished steadily as the pressure was reduced. These fluctuations were presumably related to the passage of bubbles through the pumping tube. One fact that hinders the above interpretation is the nearly constant spread of values of $\log _{e} \alpha$ over the whole isotherm, although the vapor pressures of the nitrogen rich solutions are several times those of the oxygen rich solutions. Another corroborative piece of evidence is the cooling effect of the circulation. This might be possible if evaporation were occurring near the thermometer and condensation in the inlet tube rather far separated from the thermometer, with a temperature gradient arising between these two locations. The cooling effect of circulation amounted to several hundredths of a degree. Rough calculations suggest that at a pressure of about $50 \mathrm{~mm}$, total condensation and evaporation from the surface of $300 \mathrm{~cm}^{3}$ of gas per minute would be sufficient to produce a temperature decrease of $0.05^{\circ}$ at the evaporating surface.

\section{Calculation of the Data}

The results of the measurements form three isotherms at $65^{\circ}, 70^{\circ}$, and $77.5^{\circ} \mathrm{K}$. Individual points deviate as much as $0.4^{\circ}$ from the isotherm, and therefore it was felt to be impractical to adjust one or more of the directly observed variables to find corresponding points exactly on the isotherm. Instead, the activity coefficient $\gamma$, or rather its logarithm, was computed for each point. Then the values $\log _{e} \gamma$, a rather slowly varying function of temperature, were adjusted to bring them to the isotherm. For the calculation of $\log _{e} \gamma$ the following formula was used for nitrogen:

$$
\log _{e} \gamma_{\mathrm{N}_{2}}=\log _{e} \frac{P y_{\mathrm{N}_{2}}}{P_{\mathrm{N}_{2}}^{\circ}}+\log _{e} \frac{f_{\mathrm{N}_{2}}}{P}-\log _{e} \frac{f_{\mathrm{N}_{2}}^{\circ}}{P_{\mathrm{N}_{2}}^{\circ}}-\log _{e} x_{\mathrm{N}_{2}}
$$

In this formula $P$ is the experimental pressure; $P_{\mathrm{N}_{2}}^{\circ}$ is the vapor pressure of pure nitrogen at the experimental temperature; $y_{\mathrm{N}_{2}}$ is the mole fraction of nitrogen in the vapor; $x_{\mathrm{N}_{2}}$ is the mole fraction of nitrogen in the liquid; $f_{\mathrm{N}_{2}}$ is the fugacity of nitrogen at pressure $P$ and $f_{\mathrm{N}_{2}}^{\circ}$ its fugacity at pressure $P_{\mathrm{N}_{2}}^{\circ}$. The term $\log _{e}$ $\frac{f_{\mathrm{N}_{2}}^{\circ}}{P_{\mathrm{N}}^{\circ}}$ is a correction for the nonideality of nitrogen gas in its pure state at its saturation line; and the term $\log _{e} \frac{f_{\mathrm{N}_{2}}}{P}$ is a correction for the nonideality of nitrogen gas in its mixture with oxygen at the experimental pressure. The latter term is derived on the basis of the proposal by Lewis and Randall [10] for the fugacity of a component of a gas mixture.

The fugacity correction term $\log _{e} \frac{f_{\mathrm{N}_{2}}}{P}-\log _{e} \frac{f_{\mathrm{N}_{2}}^{\circ}}{P_{\mathrm{N}_{2}}^{\circ}}$ was approximated by the term $B_{\mathrm{N}_{2}}^{\prime}\left(P-P_{\mathrm{N}_{2}}^{\circ}\right)$ in which $B_{\mathrm{N}_{2}}^{\prime}$, a function of temperature only, is related to the second virial coefficient. Similar formulas were used for the calculation of $\log _{e} \gamma_{\mathrm{O}_{2}}$. The values previously determined [7] of $\log _{e} \frac{f_{\mathrm{N}_{2}}^{\circ}}{P_{\mathrm{N}_{2}}^{\circ}}$ along the saturation line of nitrogen permitted the evaluation of $B_{\mathrm{N}_{2}}^{\prime}$ as a function of temperature. The values of $B_{\mathrm{O}_{2}}^{\prime}$ were obtained from a calculation of the second virial coefficient of oxygen, $B_{\mathrm{O}_{2}}$, by Woolley [11], and the relation of $B_{\mathrm{O}_{2}}^{\prime}=B_{\mathrm{O}_{2}} / R T$. The values of $B_{\mathrm{N}_{2}}^{\prime}$ and $B_{\mathrm{O}_{2}}^{\prime}$ at $65^{\circ}, 70^{\circ}$, and $77.5^{\circ}$ are shown in table 3 .

TABLE 3. The terms $B_{\mathrm{N}_{2}}^{\prime}$ and $B_{\mathrm{O}_{2}}^{\prime}$

\begin{tabular}{|c|c|c|}
\hline$T$ & $B_{\mathrm{N}_{2}}^{\prime}$ & $B_{\mathrm{O}_{2}}^{\prime}$ \\
\hline${ }^{\circ} K$ & atm-1 & atm -1 \\
65 & -0.0646 & -0.0839 \\
70 & -.0539 & -.0669 \\
77.5 & -.0385 & -.0491 \\
\hline
\end{tabular}

For $P_{\mathrm{N}_{2}}^{\circ}$ the vapor pressure data of Armstrong [7] were used; and for $P_{\mathrm{O}_{2}}^{\circ}$ the vapor pressure data of Hoge [9] were used. These values together with the fugacity correction terms are shown in table 1 . The terms $\log _{e} a_{\mathrm{N}_{2}}$ and $\log _{\mathrm{e}} a_{\mathrm{N}_{2}}$, in which $a$ is the activity were calculated from the preceding data for each experimental point and are also shown in table 1. The terms $\log _{\mathrm{e}} \gamma_{\mathrm{N}_{2}}$ and $\log _{\mathrm{e}} \gamma_{\mathrm{O}_{2}}$ for each run at the experimental temperature are shown in table 2. The $\Delta \log _{\mathrm{e}} \gamma$ terms are the corrections needed to bring the values of $\log _{\mathrm{e}} \gamma$ to the isotherms, and are based on the small shift in $\log _{\mathrm{e}} \gamma$ with temperature. The columns labelled $\log _{\mathrm{e}} \gamma_{\mathrm{N}_{2}}$ (at isotherm) and $\log _{\mathrm{e}} \gamma_{\mathrm{O}_{2}}$ (at isotherm) have been thus corrected for the small temperature difference existing between the observed point and the isotherm, and these values are used in subsequent calculations.

For the correlation of the data two functions were considered:

$$
\begin{aligned}
& R T \\
& V_{\mathrm{N}_{2}} \log _{e} \gamma_{\mathrm{N}_{2}}=A_{12} \phi_{\mathrm{O}_{2}}^{2} \\
& \frac{R T}{V_{\mathrm{O}_{2}}} \log _{e} \gamma_{\mathrm{o}_{2}}=A_{12} \phi_{\mathrm{N}_{2}}^{2}
\end{aligned}
$$

and

$$
\begin{aligned}
& \frac{R T}{V_{\mathrm{N}_{2}}} \log _{e} \gamma_{\mathrm{N}_{2}}=\frac{R T}{V_{\mathrm{N}_{2}}}\left[\log \frac{\phi_{\mathrm{N}_{2}}}{x_{\mathrm{N}_{2}}}+\phi_{\mathrm{O}_{2}}\left(1-\frac{V_{\mathrm{N}_{2}}}{V_{\mathrm{O}_{2}}}\right)\right]+A_{12} \phi_{\mathrm{O}_{2}}^{2} \\
& \frac{R T}{V_{\mathrm{O}_{2}}} \log _{e} \gamma_{\mathrm{O}_{2}}=\frac{R T}{V_{\mathrm{O}_{2}}}\left[\log \frac{\phi_{\mathrm{O}_{2}}}{x_{\mathrm{O}_{2}}}+\phi_{\mathrm{N}_{2}}\left(1-\frac{V_{\mathrm{O}_{2}}}{V_{\mathrm{N}_{2}}}\right)\right]+A_{12} \phi_{\mathrm{N}_{2}}^{2}
\end{aligned}
$$


In these equations $V_{\mathrm{N}_{2}}$ and $V_{\mathrm{O}_{2}}$ are the molar volumes respectively of nitrogen and oxygen; $\phi_{\mathrm{N}_{2}}$ and $\phi_{\mathrm{O}_{2}}$ are the volume fractions in the solution, $A_{12}=\left(C_{11}+C_{22}-2 C_{12}\right)$, where $C_{11}$ and $C_{22}$ are the cohesive energy densities of the pure components and $C_{12}$ results from the interaction of the two different molecules.

Equations (1a) and (1b) follow from Hildebrand's criterion of a regular solution, that the entropy of mixing is ideal. The additional terms in eq (2a) and $(2 \mathrm{~b})$ reflect the probable maximum effect of differences in molecular size on the entropy of mixing.

For the molar volumes required, the data of Baly and Donnan [12] were used for oxygen and the data of Mathias, Onnes, and Crommelin [13] were used for nitrogen. The volume fraction in these mixtures is significantly different from the mole fraction, as is shown in table 4 , in which are tabulated the volume fractions at uniform intervals of mole fractions for the three isotherms. In this calculation the assumption was made that there is no volume change in mixing.

TABLE 4. Liquid oxygen and nitrogen molar volumes and volume fractions

\begin{tabular}{|c|c|c|c|c|c|c|}
\hline \multirow{2}{*}{$x_{\mathrm{N}_{2}}$} & \multicolumn{2}{|c|}{$T=77.5^{\circ} \mathrm{K}$} & \multicolumn{2}{|c|}{$T=70^{\circ} \mathrm{K}$} & \multicolumn{2}{|c|}{$T=65^{\circ} \mathrm{K}$} \\
\hline & $V$ & $\phi_{\mathrm{N}_{2}}$ & $V$ & $\phi_{\mathrm{N}_{2}}$ & $V$ & $\phi_{N_{2}}$ \\
\hline $\begin{array}{r}0.0 \\
.1 \\
.2 \\
.3 \\
.4 \\
.5 \\
.6 \\
.7 \\
.8 \\
.9 \\
1.0\end{array}$ & $\begin{array}{c}c m^{3} \\
26.58 \\
27.40 \\
28.21 \\
29.03 \\
29.84 \\
30.66 \\
31.47 \\
32.29 \\
33.10 \\
33.92 \\
34.73\end{array}$ & $\begin{array}{r}0.0000 \\
.1268 \\
.2462 \\
.3589 \\
.4655 \\
.5664 \\
.6622 \\
.7530 \\
.8394 \\
.9216 \\
1.0000\end{array}$ & $\begin{array}{c}c m^{3} \\
25.82 \\
26.58 \\
27.34 \\
28.09 \\
28.85 \\
29.61 \\
30.37 \\
31.13 \\
31.88 \\
32.64 \\
33.40\end{array}$ & $\begin{array}{r}0.0000 \\
.1257 \\
.2443 \\
.3567 \\
.4631 \\
.5640 \\
.6599 \\
.7510 \\
.8381 \\
.9210 \\
1.0000\end{array}$ & $\begin{array}{c}c m^{3} \\
25.32 \\
26.05 \\
26.77 \\
27.50 \\
28.22 \\
28.95 \\
29.68 \\
30.40 \\
31.13 \\
31.85 \\
32.58\end{array}$ & $\begin{array}{r}0.0000 \\
.1251 \\
.2434 \\
.3554 \\
.4618 \\
.5627 \\
.6586 \\
.7502 \\
.8373 \\
.9206 \\
1.0000\end{array}$ \\
\hline
\end{tabular}

The terms $\frac{R T}{V_{\mathrm{N}_{2}}}\left[\log _{e} \frac{\phi_{\mathrm{N}_{2}}}{x_{\mathrm{N}_{2}}}+\phi_{\mathrm{O}_{2}}\left(1-\frac{V_{\mathrm{N}_{2}}}{V_{\mathrm{O}_{2}}}\right)\right]$ were evaluated at even values of $x_{\mathrm{N}_{2}}$ for the three isotherms, and when plotted against $\phi_{\mathrm{O}_{2}}^{2}$ are very nearly linear. In figure 6 are shown these functions and also $\phi_{\mathrm{O}_{2}}^{2}\left(\delta_{\mathrm{N}_{2}}-\delta_{\mathrm{O}_{2}}\right)^{2}$. In the latter term $\left(\delta_{\mathrm{N}_{2}}-\delta_{\mathrm{O}_{2}}\right)^{2}$ represents the $A_{12}$ of eq (1) and is obtained on the assumption that $C_{12}=\left(C_{11} C_{22}\right)^{1 / 2}$ and by replacing $C_{11}$ by $\delta_{\mathrm{N}_{2}}^{2}$ and $C_{22}$ by $\delta_{\mathrm{O}_{2}}^{2}$.

Thus it appears that if $\frac{R T}{V_{\mathrm{N}_{2}}} \log _{e} \gamma_{\mathrm{N}_{2}}$ is plotted as a function of $\phi_{\mathrm{O}_{2}}^{2}$, a linear relation should be obtained if either eq 1 or eq 2 apply to the system. A plot of the data is shown in figures 7,8 and 9 , in which $\frac{R T}{V_{\mathrm{N}_{2}}} \log _{e} \gamma_{\mathrm{N}_{2}}$ is plotted against $\phi_{\mathrm{C}_{2}}^{2}$ and on the same scale, by interchanging the variables, $\frac{R T}{V_{\mathrm{O}_{2}}} \log _{e} \gamma_{\mathrm{O}_{2}}$ is plotted as a function of $\phi_{\mathrm{N}_{2}}^{2}$. If eq (1) applies, the data should fall on a single straight line for each iso-

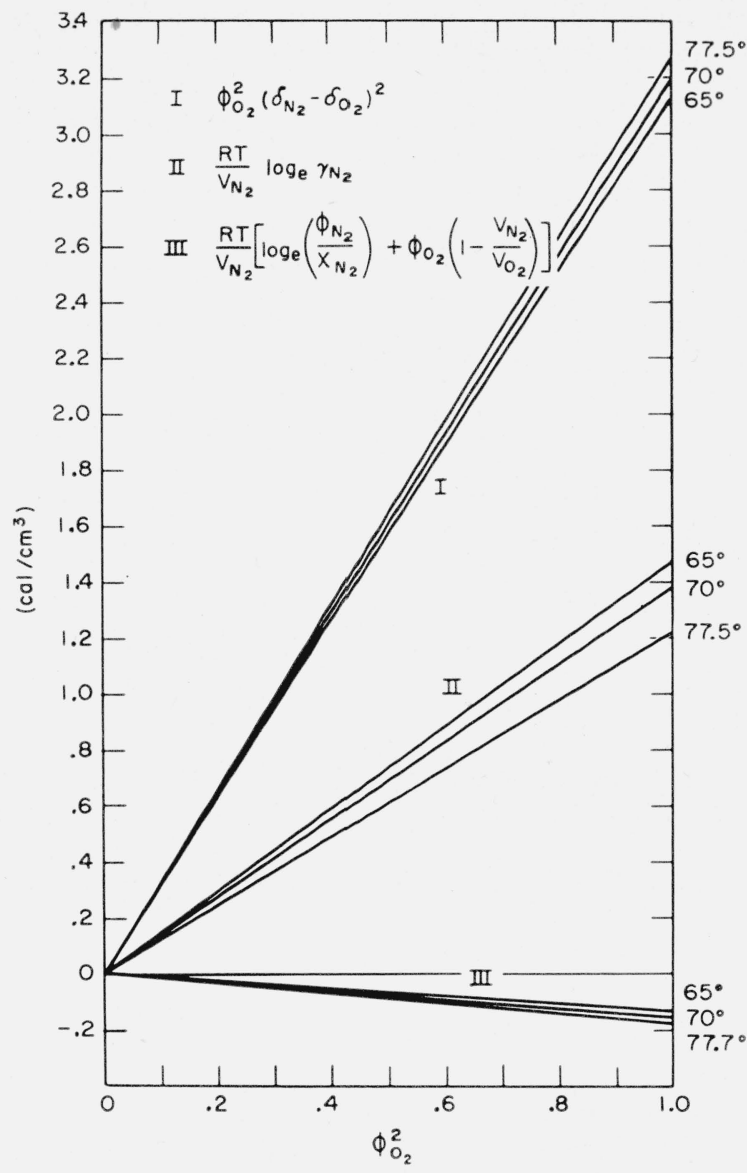

FIGURE 6. Experimental and theoretical deviations from ideality of oxygen-nitrogen solutions.

I, $\frac{R T}{V_{\mathrm{N}_{2}}} \log \gamma_{e} \gamma_{\mathrm{N}_{2}}=\phi_{\mathrm{O}_{2}}^{2}\left(\delta_{\mathrm{N}_{2}}-\delta \mathrm{O}_{2}\right)^{2}$ calculated on the basis that the solutions are regular. II, Smoothed experimental values of $\frac{R T}{V_{\mathrm{N}_{2}}} \log _{e} \gamma_{\mathrm{N}_{2}}$. III, Probable maximum contribution to non-ideality caused by differences in molar volumes.

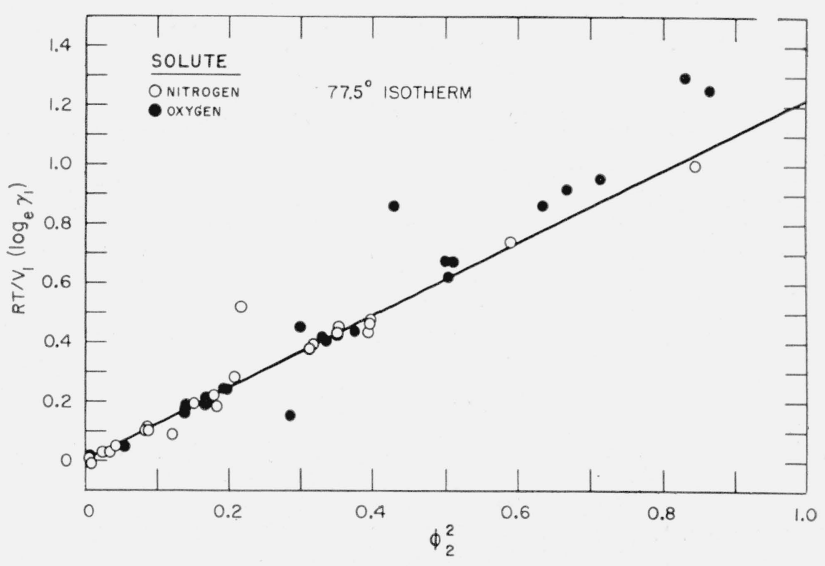

FIGURE 7. Activity coefficients in oxygen-nitrogen solutions on the $7 \% .5^{\circ}$ isotherm.

$\mathrm{O}$, Nitrogen $=1$, oxygen $=2 ; \boldsymbol{\bullet}$, oxyger $=1$; nitrogen $=2$. 


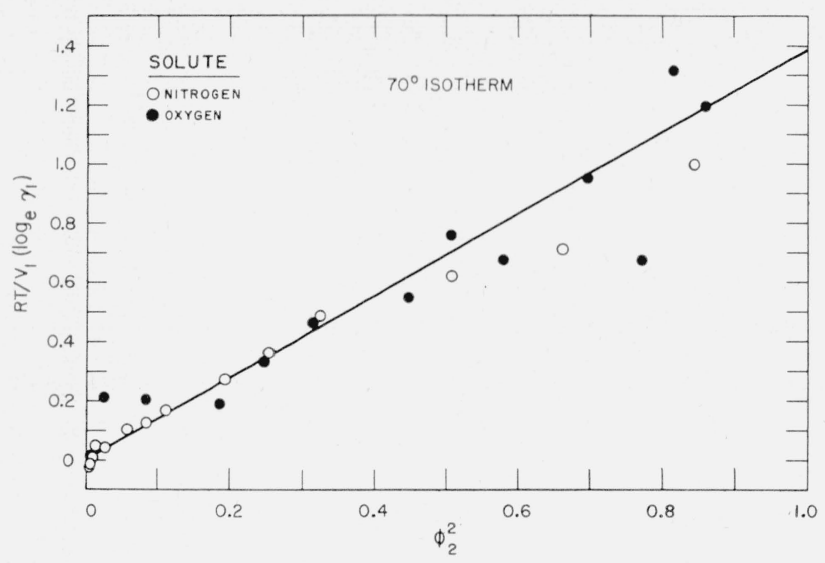

Figure 8. Activity coefficients in oxygen-nitrogen solutions on the $70^{\circ}$ isotherm.

$\bigcirc$, Nitrogen $=1$, oxygen $=2 ; \boldsymbol{\bullet}$, axygen $=1$, nitrogen $=2$.

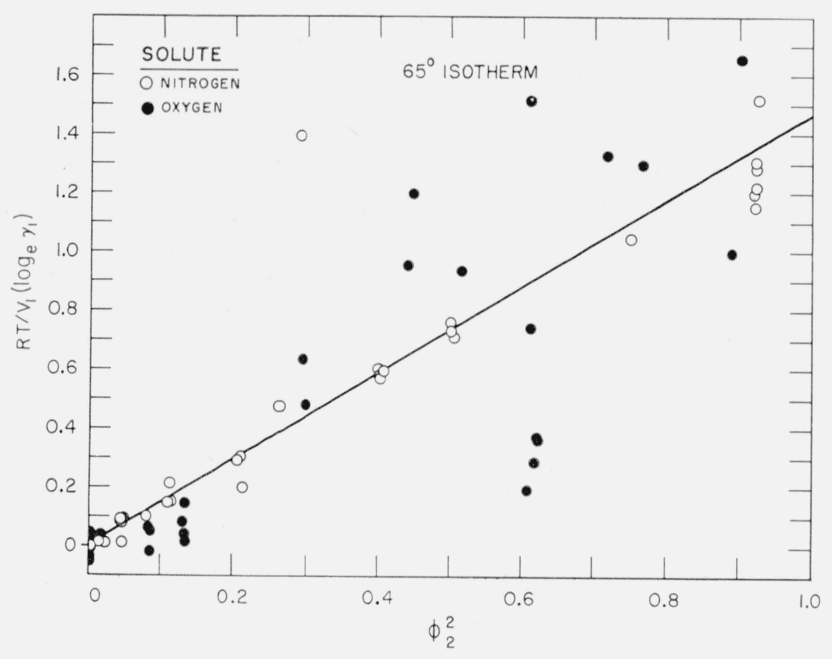

Figure 9. Activity coefficients in oxygen-nitrogen solutions on the $65^{\circ}$ isotherm.

$\bigcirc$, Nitrogen $=1$, oxygen $=2 ; \boldsymbol{\bullet}$, oxygen $=1$, nitrogen $=2$.

therm. It is seen that there is a reasonably good fit, with a fairly definite tendency for $\frac{R T}{V_{\mathrm{O}_{2}}} \log _{e} \gamma_{\mathrm{O}_{2}}$ to lie somewhat above $\frac{R T}{V_{\mathrm{N}_{2}}} \log _{e} \gamma_{\mathrm{N}_{2}}$ in the region of large $\phi_{\mathrm{N}_{2}}$ In passing a line through the data, rather large weight was given to the values of $\frac{R T}{V_{\mathrm{N}_{2}}} \log _{\ell} \gamma_{\mathrm{N}_{2}}$ because these points show very much less scatter than do the $\frac{R T}{V_{\mathrm{O}_{2}}} \log _{e} \gamma_{\mathrm{O}_{2}}$ points.

The separation coefficient $\alpha=\frac{y_{\mathrm{N}_{2}} / y_{\mathrm{O}_{2}}}{x_{\mathrm{N}_{2}} / x_{\mathrm{O}_{2}}}$ was also calculated for each experimental point and is shown in column 9 of table 1. $\log _{e} \alpha$ is related to $\log _{e} \gamma_{\mathrm{N}_{2}}$ and $\log _{e} \gamma_{\mathrm{O}_{2}}$ by eq (3)

$\log _{e} \gamma_{\mathrm{N}_{2}} / \gamma_{\mathrm{O}_{2}}=\log _{e} \alpha+\log _{e} P_{\mathrm{O}_{2}}^{\circ}-\log _{e} P_{\mathrm{N}_{2}}^{\circ}$

$$
+\mathrm{B}_{\mathrm{N}_{2}}^{\prime}\left(P-P_{\mathrm{N}_{2}}^{\circ}\right)-\mathrm{B}_{\mathrm{O}_{2}}^{\prime}\left(P-P_{\mathrm{O}_{2}}^{\circ}\right)
$$

If $\log _{e} \gamma_{\mathrm{N}_{2}}$ and $\log _{e} \gamma \mathrm{O}_{2}$ are replaced by $V_{\mathrm{N}_{2}}\left(\frac{A_{12}}{R T}\right) \phi_{\mathrm{O}_{2}}^{2}$ and $V_{\mathrm{O}_{2}}\left(\frac{A_{12}}{R T}\right) \phi_{\mathrm{N}_{2}}^{2}$, then it is possible to devise a smooth function for $\log _{e} \alpha$ for each isotherm, which is consistent with the activity data. The smooth functions $\log _{e} \alpha$ are shown in figure 5 as the solid lines. The variation of $\log _{e} \alpha$ with temperature can be used to bring exactly to the isotherm the values of $\log _{e} \alpha$ calculated at the experimental temperatures, and this has been done, assuming for this purpose that over the range of the temperature correction $\log _{e} \alpha$ varies linearly with temperature. The corrections were made at constant $\phi_{\mathrm{N}_{2}}$. The change in $x_{\mathrm{N}_{2}}$ at constant $\phi_{\mathrm{N}_{2}}$ is less than 0.0002 for the largest corrections in temperatures that were made, and so changes in $x_{\mathrm{N}_{2}}$ have not been made. The corrected values of $\log _{e} \alpha$ are shown in figure 5, plotted against the mole fractions of nitrogen in the liquid. It will be noted that in this graph there is a tendency for the data at the extreme ends to fall below the solid curves, suggesting that a straighter line would fit somewhat better. The tendency to fall below is perhaps related to the tendency of the values of $\frac{R T}{V_{\mathrm{O}_{2}}} \log _{e} \gamma_{\mathrm{O}_{2}}$ to lie above the fitted lines in the plot against $\phi_{\mathrm{N}_{2}}^{2}$. However, no straight line function of $\frac{R T}{V_{1}} \log _{e} \gamma_{1}$ against $\phi_{2}^{2}$ can be devised which will cause $\log _{e} \alpha$ to fit the data at both ends.

The calculation of the equilibrium vapor composition for a given liquid composition can easily be made using the smooth values of $\log _{e} \alpha$. It is also then possible to calculate the partial pressure of each component and the total vapor pressure of the solution for any value of $y_{\mathrm{N}_{2}}$ or $x_{\mathrm{N}_{2}}$ using the smoothed values of $\log _{e} \gamma_{\mathrm{N}_{2}}$ in eq (4) and $\log _{e} \gamma_{\mathrm{O}_{2}}$ in a similar equation.

$\log _{e} \gamma_{\mathrm{N}_{2}}=\log _{e} P+\log _{e} y_{\mathrm{N}_{2}}+\log _{e}(f / P)_{\mathrm{N}_{2}}$

$$
-\log _{e} P_{\mathrm{N}_{2}}^{\circ}-\log _{e}\left(f^{\circ} / P^{\circ}\right)_{\mathrm{N}_{2}}-\log _{e} x_{\mathrm{N}_{2}}
$$

\section{Calculation of Cohesive Energy Densities for Oxygen and Nitrogen}

Following Hildebrand and Scott [14], consider the cohesive energy density $C_{11}$ to be $-\frac{E}{V_{l}}$ where $V_{l}$ is the molar volume of the liquid and $E$ is the energy required to evaporate one mole of liquid to vapor at zero pressure. E may be obtained from the heat 
of vaporization by the following relation $-E=$ $\left[\Delta H_{\text {vap }}-P\left(V_{g}-V_{l}\right)\right] \frac{V_{g}}{V_{g}-V_{l}}$ in which $\Delta H_{\text {vap }}$ is the latent heat of vaporization to the vapor at saturation pressure $P$, and $V_{g}$ is the molar volume of vapor. The factor $\frac{V_{g}}{V_{g}-V_{l}}$ is required to account for the energy change on reducing the pressure of the vapor to zero. For this calculation of $-E$ we were able to use recent values for the $\Delta H_{\text {vap }}$ of oxygen determined by Furukawa and McCoskey [15]. The values used are shown in the second column of table 5 . The corresponding vapor pressures for oxygen, shown in the third column, were taken from Hoge [9] and those for nitrogen were calculated from the equation given by Armstrong [7]. Vapor volumes were calculated from the same vapor pressure sources by the use of the relation $\left(V_{g}-V_{l}\right)=\Delta H_{\mathrm{vap}} / T \frac{d P}{d T}$. As elsewhere in this work the liquid volumes of oxygen were determined from the values reported by Baly and Donnan [12], and those of nitrogen were determined from the values reported by Mathias, Onnes, and Crommelin [13]. The cohesive energy density is shown in the ninth column of table 5 ; and its square root, the solubility parameter $\delta$, is shown in the last column. The solubility parameter for nitrogen, $\delta_{\mathrm{N}_{2}}$, is a linear function of temperature and may be represented by eq (5)

$$
\delta_{\mathrm{N}_{2}}=8.7516-0.03720 T
$$

with a difference no greater than 0.001 from the tabulated values of $\delta$. The solubility parameter for oxygen, $\delta_{\mathrm{O}_{2}}$, is nearly linear with temperature, but a small deviation, which will be called $\Delta$, is notice. able. Values of $\delta_{\mathrm{O}_{2}}$ may be interpolated using eq (6)

$$
\delta_{\mathrm{O}_{2}}=10.3666-0.03476 T+\Delta
$$

( $\Delta$ is zero at $68.40^{\circ}$ and $91.30^{\circ} \mathrm{K}$ and has the values + 0.0052 at $76.00^{\circ}$ and +0.0047 at $84.10^{\circ} \mathrm{K}$ ) in which the value of $\Delta$ may be selected with sufficient accuracy from a smooth curve drawn through the points noted with the equation.

\section{Comparison of Experimental and Theoretical Results}

Using the values of $\delta_{\mathrm{O}_{2}}$ and $\delta_{\mathrm{N}_{2}}$ calculated in section 5, the terms $\phi_{\mathrm{O}_{2}}^{2}\left(\delta_{\mathrm{N}_{2}}-\delta_{\mathrm{O}_{2}}\right)^{2}$ have been calculated for the three isotherms and are shown as the group I of lines in figure 6. For comparison the terms $\frac{R T}{V_{\mathrm{N}_{2}}} \log _{e} \gamma_{\mathrm{N}_{2}}$ obtained from the experimental data are also shown as group II of lines. Group III lines are the term $\frac{R T}{V_{\mathrm{N}_{2}}}\left[\log _{e} \frac{\phi_{\mathrm{N}_{2}}}{x_{\mathrm{N}_{2}}}+\phi_{\mathrm{O}_{2}}\left(1-\frac{V_{\mathrm{N}_{2}}}{V_{\mathrm{O}_{2}}}\right)\right]$. It is seen that they are very small compared to either of the other functions or to the difference between them, and so will not be considered further. The intercepts of the group I lines at $\phi_{\mathrm{O}_{2}}^{2}=1$ are the experimental values of $A_{12}$ for the three temperatures. If $A_{12}$ were a true constant the isotherms would be superimposed in this plot; but as it is there is a slight increase of $A_{12}$ as the temperature is reduced. One assumption made in deriving the theoretical function $\frac{R T}{V_{\mathrm{N}_{2}}} \log _{e} \gamma_{\mathrm{N}_{2}}=\phi_{\mathrm{O}_{2}}^{2}\left(\delta_{\mathrm{N}_{2}}-\delta_{\mathrm{O}_{2}}\right)^{2}$ is that the term $C_{12}$ is the geometric mean of $C_{11}$ and $C_{22}$. The constant $A_{12}$ becomes $\left(\delta_{\mathrm{N}_{2}}-\delta_{\mathrm{O}_{2}}\right)^{2}$ and its value at $77.5^{\circ}$ becomes $3.27 \mathrm{cal} / \mathrm{cm}^{3}$ as compared to $1.22 \mathrm{cal} / \mathrm{cm}^{3}$ derived from the experimental data. An assumption that $C_{12}=\frac{C_{11}+C_{22}}{2}$ would lead to $A_{12}=0$. Thus the conclusion seems justified that the interaction energies between unlike molecules lie somewhere between the arithmetic and geometric mean of the interactions between like mole-

TABLE 6. Experimental and theoretical interaction energies of oxygen and nitrogen

\begin{tabular}{|c|c|c|c|c|c|c|c|}
\hline$T$ & $C_{11^{\mathrm{a}}}$ & $C_{22}$ & $\left(\delta_{1}-\delta_{2}\right)^{2}$ & $A_{12}(\operatorname{expt})$ & $C_{12}(\operatorname{expt})$ & $\left(C_{11} C_{22}\right)^{1 / 2}$ & $1 / 2\left(C_{11}+C_{22}\right)$ \\
\hline $65^{\circ}$ & 40.12 & 65.69 & 3. 14 & 1.47 & 52.17 & 51. 34 & 52.91 \\
\hline $70^{\circ}$ & 37.80 & 62.97 & 3. 19 & 1.38 & 49.70 & 48. 78 & 50.39 \\
\hline $77.5^{\circ}$ & 34.44 & 58.95 & 3. 27 & 1. 22 & 46.09 & 45.06 & 46.70 \\
\hline
\end{tabular}

$\left(\mathrm{cal} \mathrm{cm}^{-3}\right)$

a Subscript 1 refers to nitrogen, 2 refers to oxygen.

\begin{tabular}{|c|c|c|c|c|c|c|c|c|c|}
\hline $\mathrm{T}$ & $\Delta H_{v}$ & $P$ & $V_{g}$ & $V_{l}$ & $\frac{\Delta H V_{g}}{V_{g}-V_{l}}$ & $P V_{g}$ & $-E$ & $\frac{-E}{V_{l}}$ & $\delta$ \\
\hline \multicolumn{10}{|c|}{ Oxygen } \\
\hline $\begin{array}{c}{ }^{\circ} K \\
68.40 \\
76.00 \\
84.10 \\
91.30\end{array}$ & $\begin{array}{c}c a l \\
\text { mole } \\
1773.0 \\
1727.6 \\
1674.2 \\
1622.9\end{array}$ & $\begin{array}{c}m m \mathrm{Hg} \\
34.70 \\
126.74 \\
381.6 \\
852.3\end{array}$ & $\begin{array}{c}\mathrm{cm}^{3} \\
\text { 1. } 228 \times 10^{5} \\
\text { 3. } 707 \times 10^{4} \\
\text { 1. } 344 \times 10^{4} \\
\text { 6. } 420 \times 10^{3}\end{array}$ & $\begin{array}{c}\mathrm{cm}^{3} \\
25.66 \\
26.42 \\
27.30 \\
28.12\end{array}$ & $\begin{array}{c}\text { cal mole-1 } \\
1773.4 \\
1728.8 \\
1677.7 \\
1630.0\end{array}$ & $\begin{array}{c}\text { cal mole-1 } \\
135.8 \\
149.7 \\
163.4 \\
175.2\end{array}$ & $\begin{array}{c}c a l \\
\text { mole } \\
1637.6 \\
1579.1 \\
1514.3 \\
1454.8\end{array}$ & $\begin{array}{c}\text { cal mole-1 } \\
\mathrm{cm}^{-3} \\
63.82 \\
59.77 \\
55.47 \\
51.74\end{array}$ & $\begin{array}{l}7.989 \\
7.731 \\
\text { 7. } 448 \\
7.193\end{array}$ \\
\hline \multicolumn{10}{|c|}{ Nitrogen } \\
\hline $\begin{array}{l}68.00 \\
73.10 \\
77.364 \\
78.00\end{array}$ & $\begin{array}{l}1409.9 \\
1370.7 \\
1336.8 \\
1331.7\end{array}$ & $\begin{array}{l}213.54 \\
445.77 \\
760.00 \\
818.48\end{array}$ & $\begin{array}{l}\text { 1. } 954 \times 10^{4} \\
\text { 9. } 945 \times 10^{3} \\
\text { 6. } 100 \times 10^{3} \\
\text { 5. } 700 \times 10^{3}\end{array}$ & $\begin{array}{l}33.05 \\
33.92 \\
34.70 \\
34.81\end{array}$ & $\begin{array}{l}1412.3 \\
1375.4 \\
1344.4 \\
1339.9\end{array}$ & $\begin{array}{l}132.9 \\
141.3 \\
147.7 \\
148.7\end{array}$ & $\begin{array}{l}1279.4 \\
1234.1 \\
1196.7 \\
1191.2\end{array}$ & $\begin{array}{l}\text { 38. } 71 \\
36.38 \\
34.49 \\
34.22\end{array}$ & $\begin{array}{l}\text { 6. } 222 \\
6.032 \\
5.873 \\
5.850\end{array}$ \\
\hline
\end{tabular}

TARLE 5. Cohesive energy densities of oxygen and nit:ogen 
cules of the two species. Table 6 shows $C_{11}$ and $C_{22}$ at the three isotherms, evaluated by interpolation from the figures in table 5 , and the value of $C_{12}$ calculated from the experiments and compared with $\left(C_{11} C_{22}\right)^{1 / 2}$ and $\frac{1}{2}\left(C_{11}+C_{22}\right)$. In this table subscript 1 refers to nitrogen and subscript 2 refers to oxygen.

The solutions of oxygen and nitrogen, when corrected for the nonideal behavior of the vapors, are considerably more nearly ideal than would be predicted on the basis of the geometric mean hypothesis of interactions. Any possible tendency of oxygen to form dimers at low temperatures would result in positive deviations from Raoult's law. Because the deviations observed are extremely small, and are much smaller than might be expected even in the absence of such dimerization, it seems unreasonable to attribute any of the observed solution properties to such behavior.

When a plot is made, as in figures 7,8 , and 9 , of $\frac{R T}{V_{1}} \log _{e} \gamma_{1}$ against volume fraction of component 2 , the nitrogen activity data at the $77.5^{\circ}$ isotherm appears to form a good straight line, but the oxygen activities tend to curve upward. On the other hand, when plotted against mole fraction squared, the $\log _{e} \gamma_{\mathrm{N}_{2}}$ curves definitely upward and $\log _{e} \gamma_{\mathrm{O}_{2}}$ curves downward. This suggests that while the volume fraction is a more accurate guide to the solution behavior, the solutions behave as though the molar volumes in solution are more nearly equal than would be expected from the molar volumes of the pure components.

A similar interpretation appears to be suggested by the plots of $\log _{e} \alpha$ against $x_{N_{2}}$. Here, if the molar volumes were equal, a straight line would be ex- pected, as contrasted with the curved line calculated on the basis of the different molar volumes of the pure components. There is a definite tendency, as noted in section 4 , for the experimental points at the extreme ends to fall below the solid curves, suggesting that straight lines would fit the data almost as well as the curved lines shown.

\section{References}

[1] E. C. C. Baly, Phil. Mag. [5] 49, 517 (1900).

[2] J. K. H. Inglis, Phil. Mag. [6] 11, 640 (1906).

[3] B. F. Dodge and A. K. Dunbar, J. Am. Chem. Soc. 49, 591 (1927).

[4] O. N. Trapeznikova and L. V. Shubnikov, J. Tech. Phys. (U. S. S. R.) 5, 949 (1934).

[5] M. L. Sagenkahn and H. L. Fink, Office of Scientific Research and Development Report 4493 (Dec. 22, 1944).

[6] H. J. Hoge and F. G. Brickwedde, J. Research NBS 22, 351 (1923) RP1188.

[7] G. T. Armstrong, J. Research NBS 53, 263 (1954) RP2543.

[8] M. V. Kilday, J. Research NBS 45, 43 (1950) RP 2112.

[9] H. J. Hoge, J. Research NBS 44, 321 (1950) RP2081.

[10] G. N. Lewis and M. Randall, Thermodynamics, pp. 225-7, (McGraw-Hill Book Co., Inc., New York, N. Y., 1923).

[11] H. W. Woolley (private communication).

[12] E. C. C. Baly and F. G. Donnan, J. Chem. Soc. 81, 907 (1902).

[13] E. Mathias, H. K. Onnes and C. A. Crommelin, Koninkl Akad. Wetenschap. Amsterdam 17, 953 (1915).

[14] J. H. Hildebrand and R. L. Scott, The solubility of nonelectrolytes, 3rd ed., ch. XXIII, (Reinhold Publishing Corp., New York, N. Y., 1950).

[15] G. T. Furukawa and R. E. McCoskey (private communication).

Washington, June 16, 1955. 